Moroccan J. of Pure and Appl. Anal. (MJPAA)

Volume 7(1), 2021, Pages 134-172

ISSN: Online 2351-8227 - Print 2605-6364

DOI: $10.2478 / \mathrm{mjpaa}-2021-0012$

\title{
Complete Approximations by Multivariate Generalized Gauss-Weierstrass Singular Integrals
}

\author{
George A. Anastassiou ${ }^{1}$
}

Aвstract. This research and survey article deals exclusively with the study of the approximation of generalized multivariate Gauss-Weierstrass singular integrals to the identity-unit operator. Here we study quantitatively most of their approximation properties. The multivariate generalized Gauss-Weierstrass operators are not in general positive linear operators. In particular we study the rate of convergence of these operators to the unit operator, as well as the related simultaneous approximation. These are given via Jackson type inequalities and by the use of multivariate high order modulus of smoothness of the high order partial derivatives of the involved function. Also we study the global smoothness preservation properties of these operators. These multivariate inequalities are nearly sharp and in one case the inequality is attained, that is sharp. Furthermore we give asymptotic expansions of Voronovskaya type for the error of multivariate approximation. The above properties are studied with respect to $L_{p}$ norm, $1 \leq p \leq \infty$.

Mathematics Subject Classification (2020). 26A15, 26D15, 41A17, 41A35.

Key words and phrases. Approximations by multivariate Gauss-Weierstrass singular integral, Multivariate moduli of smoothness.

\section{Introduction}

We start with our motivation for this work which comes from [6].

In the next we introduce and deal with the smooth Gauss-Weierstrass singular integral operators $W_{r, \xi}(f, x)$ defined as follows.

Received : October 10, 2020 - Accepted: November 25, 2020.

(C)The Author(s) 2020. This article is published with open access by Sidi Mohamed Ben Abdallah University.

${ }^{1}$ Department of Mathematical Sciences University of Memphis Memphis, TN 38152, U.S.A.

e-mail ${ }^{1}$ :ganastss@memphis.edu. 
For $r \in \mathbb{N}$ and $n \in \mathbb{Z}_{+}$we set

$$
\alpha_{j}=\left\{\begin{array}{l}
(-1)^{r-j}\left(\begin{array}{c}
r \\
j
\end{array}\right) j^{-n}, j=1, \ldots, r, \\
1-\sum_{j=1}^{r}(-1)^{r-j}\left(\begin{array}{c}
r \\
j
\end{array}\right) j^{-n}, j=0
\end{array}\right.
$$

that is $\sum_{j=0}^{r} \alpha_{j}=1$.

Let $f \in C^{n}(\mathbb{R})$ and $f^{(n)} \in L_{p}(\mathbb{R}), 1 \leq p<\infty$, we define for $x \in \mathbb{R}, \xi>0$ the Lebesgue integral

$$
W_{r, \xi}(f ; x)=\frac{1}{\sqrt{\pi \xi}} \int_{-\infty}^{\infty}\left(\sum_{j=0}^{r} \alpha_{j} f(x+j t)\right) e^{-\frac{t^{2}}{\zeta}} d t
$$

Note 1.1. The operators $W_{r, \xi}$ are not, in general, positive.

Let $r=2, n=3$. Then $\alpha_{0}=\frac{23}{8}, \alpha_{1}=-2, \alpha_{2}=\frac{1}{8}$. Consider $f(t)=t^{2} \geq 0$ and $x=0$. Then

$$
W_{r, \xi}\left(t^{2} ; 0\right)=-\frac{3 \xi}{4}<0
$$

We notice by $\frac{1}{\sqrt{\pi \xi}} \int_{-\infty}^{\infty} e^{-\frac{t^{2}}{\zeta}} d t=1$, that $W_{r, \xi}(c, x)=c, c$ constant, and

$$
W_{r, \xi}(f ; x)-f(x)=\frac{1}{\sqrt{\pi \xi}}\left(\sum_{j=0}^{r} \alpha_{j} \int_{-\infty}^{\infty}(f(x+j t)-f(x)) e^{-\frac{t^{2}}{\xi}} d t\right) .
$$

We need the $r$ th $L_{p^{-}}$modulus of smoothness

$$
\omega_{r}\left(f^{(n)}, h\right)_{p}:=\sup _{|t| \leq h}\left\|\Delta_{t}^{r} f^{(n)}(x)\right\|_{p, x^{\prime}}, h>0,
$$

where

$$
\Delta_{t}^{r} f^{(n)}(x):=\sum_{j=0}^{r}(-1)^{r-j}\left(\begin{array}{l}
r \\
j
\end{array}\right) f^{(n)}(x+j t),
$$

see [8], p. 44. Here we have that $\omega_{r}\left(f^{(n)}, h\right)_{p}<\infty, h>0$.

We need to introduce

$$
\delta_{k}:=\sum_{j=1}^{r} \alpha_{j} j^{k}, \quad k=1, \ldots, n \in \mathbb{N}
$$

and denote by $\lfloor\cdot\rfloor$ the integral part. Call

$$
r(w, x):=\sum_{j=0}^{r} \alpha_{j} j^{n} f^{(n)}(x+j w)-\delta_{n} f^{(n)}(x) .
$$

According to [2], p. 306, [1], we get

$$
\tau(w, x)=\Delta_{w}^{r} f^{(n)}(x) .
$$


Thus

$$
\|\tau(w, x)\|_{p, x} \leq w_{r}\left(f^{(n)},|w|\right)_{p^{\prime}}, w \in \mathbb{R}
$$

Define

$$
R_{n}(0, t, x):=\int_{0}^{t} \frac{(t-w)^{n-1}}{(n-1) !} \tau(w, x) d w, \quad n \in \mathbb{N} .
$$

Using the above terminology ([6]) we obtain

$$
\begin{gathered}
\Delta(x):=W_{r, \xi}(f ; x)-f(x)- \\
\sum_{m=1}^{\left\lfloor\frac{n}{2}\right\rfloor} f^{(2 m)}(x) \delta_{2 m} \frac{(2 m-1)(2 m-3) \cdot \ldots \cdot 3 \cdot 1}{(2 m) !}\left(\frac{\xi}{2}\right)^{m}=R_{n}^{*}(x),
\end{gathered}
$$

where

$$
R_{n}^{*}(x):=\frac{1}{\sqrt{\pi \xi}} \int_{-\infty}^{\infty} R_{n}(0, t, x) e^{-\frac{t^{2}}{\zeta}} d t, \quad n \in \mathbb{N} .
$$

In $\Delta(x)$, see (11), the sum collapses when $n=1$.

We mention

Theorem 1.2. ([6]) Let $p, q>1$ such that $\frac{1}{p}+\frac{1}{q}=1, n \in \mathbb{N}$ and the rest as above. Then

$$
\|\Delta(x)\|_{p} \leq \frac{2^{\frac{p+1}{2 p}} \tau^{\frac{1}{p}} \xi^{\frac{n}{2}}}{q^{\frac{1}{2 q}} \pi^{\frac{1}{2 p}}(r p+1)^{\frac{1}{p}}((n-1) !)(q(n-1)+1)^{\frac{1}{q}}} \omega_{r}\left(f^{(n)}, \sqrt{\xi}\right)_{p^{\prime}}
$$

where

$$
\tau:=\left[\int_{0}^{\infty}(1+u)^{r p+1} u^{n p-1} e^{-\frac{p u^{2}}{2}} d u-\int_{0}^{\infty} u^{n p-1} e^{-\frac{p u^{2}}{2}} d u\right]<\infty .
$$

Hence as $\xi \rightarrow 0$ we obtain $\|\Delta(x)\|_{p} \rightarrow 0$.

If additionally $f^{(2 m)} \in L_{p}(\mathbb{R}), m=1,2, \ldots,\left\lfloor\frac{n}{2}\right\rfloor$ then $\left\|W_{r, \xi}(f)-f\right\|_{p} \rightarrow 0$, as $\xi \rightarrow 0$.

The counterpart of Theorem 1.2 follows, case of $p=1$.

Theorem 1.3. ([6]) Let $f \in C^{n}(\mathbb{R})$ and $f^{(n)} \in L_{1}(\mathbb{R}), n \in \mathbb{N}$. Then

$$
\begin{gathered}
\|\Delta(x)\|_{1} \leq \frac{2}{(r+1)(n-1) ! \sqrt{\pi}} \\
\left(\sum_{k=1}^{r+1}\left(\begin{array}{c}
r+1 \\
k
\end{array}\right)\left(\prod_{r=1}^{\left\lfloor\frac{n+k}{2}\right\rfloor-1}(n+k-2 r)\right) \cdot M \cdot\left(\frac{1}{2}\right)^{\left\lfloor\frac{n+k}{2}\right\rfloor}\right) \omega_{r}\left(f^{(n)}, \sqrt{\xi}\right)_{1} \xi^{\frac{n}{2}}
\end{gathered}
$$

where

$$
M=\left\{\begin{array}{l}
\frac{\sqrt{\pi}}{2}, n+k \text { is even } \\
1, n+k \text { is odd }
\end{array} .\right.
$$

Hence as $\xi \rightarrow 0$ we obtain $\|\Delta(x)\|_{1} \rightarrow 0$.

If additionally $f^{(2 m)} \in L_{1}(\mathbb{R}), m=1,2, \ldots,\left\lfloor\frac{n}{2}\right\rfloor$ then $\left\|W_{r, \xi}(f)-f\right\|_{1} \rightarrow 0$, as $\xi \rightarrow 0$. 
The case $n=0$ is mentioned next.

Proposition 1.1. ([6]) Let $p, q>1$ such that $\frac{1}{p}+\frac{1}{q}=1$ and the rest as above. Then

$$
\left\|W_{r, \zeta}(f)-f\right\|_{p} \leq \frac{2^{\frac{1}{2 q}+\frac{1}{p}} \pi^{\frac{1}{2 q}-\frac{1}{2}}}{q^{\frac{1}{2 q}}} \theta^{\frac{1}{p}} \omega_{r}(f, \sqrt{\xi})_{p},
$$

where

$$
\theta:=\int_{0}^{\infty}(1+t)^{r p} e^{-\frac{p t^{2}}{2}} d t<\infty
$$

Hence as $\xi \rightarrow 0$ we obtain $W_{r, \xi}(f) \rightarrow$ unit operator $I$ in the $L_{p}$ norm, $p>1$.

We also mention

Proposition 1.2. ([6]) It holds

$$
\left\|W_{r, \xi} f-f\right\|_{1} \leq \frac{2}{\sqrt{\pi}}\left(\int_{0}^{\infty}(1+t)^{r} e^{-t^{2}} d t\right) \omega_{r}(f, \sqrt{\xi})_{1} .
$$

Hence as $\xi \rightarrow 0$ we get $W_{r, \xi} \rightarrow I$ in the $L_{1}$ norm.

In this work we study the approximation properties of multivariate smooth general GaussWeierstrass singular integral operators:

$$
\begin{gathered}
W_{r, n}^{[m]}\left(f ; x_{1}, \ldots, x_{N}\right):= \\
\frac{1}{\left(\sqrt{\pi \xi_{n}}\right)^{N}} \sum_{j=0}^{r} \alpha_{j, r}^{[m]} \int_{\mathbb{R}^{N}} f\left(x_{1}+s_{1} j, x_{2}+s_{2} j, \ldots, x_{N}+s_{N} j\right) e^{-\frac{\left(\sum_{i=1}^{N} s_{i}^{2}\right)}{\xi_{n}}} d s_{1} \ldots d s_{N} .
\end{gathered}
$$

Notice that

$$
\frac{1}{\left(\sqrt{\pi \xi_{n}}\right)^{N}} \int_{\mathbb{R}^{N}} e^{-\frac{\left(\sum_{i=1}^{N} s_{i}^{2}\right)}{\tilde{\zeta}^{2} n}} d s_{1} \ldots d s_{N}=1
$$

see [5].

Here $r \in \mathbb{N}, m \in Z_{+}$, and

$$
\alpha_{j, r}^{[m]}:=\left\{\begin{array}{l}
(-1)^{r-j}\left(\begin{array}{c}
r \\
j
\end{array}\right) j^{-m}, \quad \text { if } j=1,2, \ldots, r, \\
1-\sum_{i=1}^{r}(-1)^{r-i}\left(\begin{array}{c}
r \\
i
\end{array}\right) i^{-m}, \text { if } j=0,
\end{array}\right.
$$

and

$$
\delta_{k, r}^{[m]}:=\sum_{j=1}^{r} \alpha_{j, r}^{[m]} j^{k}, \quad k=1,2, \ldots, m \in \mathbb{N} .
$$

See that $\sum_{j=0}^{r} \alpha_{j, r}^{[m]}=1$. 
Also here $\xi_{n} \in(0,1], n \in \mathbb{N}$, and $f: \mathbb{R}^{N} \rightarrow \mathbb{R}$ is a Borel measurable function. The above operator $W_{r, n}^{[m]}$ is a special case of a more general operator $\theta_{r, n}^{[m]}$ studied in general in [3] by the author.

We mention next about $\theta_{r, n}^{[m]}$.

Let $\mu_{\xi_{n}}$ be a probability Borel measure on $\mathbb{R}^{N}, N \geq 1$.

We define the multiple smooth singular integral operators

$$
\theta_{r, n}^{[m]}\left(f ; x_{1}, \ldots, x_{N}\right):=\sum_{j=0}^{r} \alpha_{j . r}^{[m]} \int_{\mathbb{R}^{N}} f\left(x_{1}+s_{1} j, x_{2}+s_{2} j, \ldots, x_{N}+s_{N} j\right) d \mu_{\xi_{n}}(s),
$$

where $s:=\left(s_{1}, \ldots, s_{N}\right), x:=\left(x_{1}, \ldots, x_{N}\right) \in \mathbb{R}^{N}$.

The operators $\theta_{r, n}^{[m]}$ are not in general positive. For example, consider the function $\varphi\left(u_{1}, \ldots, u_{N}\right)=$ $\sum_{i=1}^{N} u_{i}^{2}$ and also take $r=2, m=3 ; x_{i}=0, i=1, \ldots, N$. See that $\varphi \geq 0$, however

$$
\begin{gathered}
\theta_{2, n}^{[3]}(\varphi ; 0,0, \ldots, 0)=\left(\sum_{j=1}^{2} j^{2} \alpha_{j, 2}^{[3]}\right) \int_{\mathbb{R}^{N}}\left(\sum_{i=1}^{N} s_{i}^{2}\right) d \mu_{\xi_{n}}(s)= \\
\left(\alpha_{1,2}^{[3]}+4 \alpha_{2,2}^{[3]}\right) \int_{\mathbb{R}^{N}}\left(\sum_{i=1}^{N} s_{i}^{2}\right) d \mu_{\xi_{n}}(s)=\left(-2+\frac{1}{2}\right) \int_{\mathbb{R}^{N}}\left(\sum_{i=1}^{N} s_{i}^{2}\right) d \mu_{\xi_{n}}(s)<0 .
\end{gathered}
$$

assuming that $\int_{\mathbb{R}^{N}}\left(\sum_{i=1}^{N} s_{i}^{2}\right) d \mu_{\xi_{n}}(s)<\infty$.

Clearly in the case of $W_{r, n}^{[m]}$ we have that

$$
d \mu_{\xi_{n}}(s)=\frac{1}{\left(\sqrt{\pi \xi_{n}}\right)^{N}} e^{-\frac{\left(\sum_{i=1}^{N} s_{i}^{2}\right)}{\xi_{n}}} d s_{1} \ldots d s_{N}, \quad s \in \mathbb{R}^{N}
$$

Lemma 1.1. The operator $\theta_{r, n}^{[m]}$ preserve the constant functions in $N$ variables.

We need

Definition 1.1. Let $f \in C_{B}\left(\mathbb{R}^{N}\right)$, the space of all bounded and continuous functions or uniformly continuous on $\mathbb{R}^{N}$. Then, the rth multivariate modulus of smoothness of $f$ is given by (see, e.g. [4])

$$
\omega_{r}(f ; h):=\sup _{\sqrt{u_{1}^{2}+\ldots+u_{N}^{2}} \leq h}\left\|\Delta_{u_{1}, u_{2}, \ldots, u_{N}}^{r}(f)\right\|_{\infty}<\infty, \quad h>0,
$$

where $\|\cdot\|_{\infty}$ is the sup-norm and

$$
\begin{gathered}
\Delta_{u}^{r} f(x):=\Delta_{u_{1}, u_{2}, \ldots, u_{N}}^{r} f\left(x_{1}, \ldots, x_{N}\right)= \\
\sum_{j=0}^{r}(-1)^{r-j}\left(\begin{array}{c}
r \\
j
\end{array}\right) f\left(x_{1}+j u_{1}, x_{2}+j u_{2}, \ldots, x_{N}+j u_{N}\right) .
\end{gathered}
$$

Let $m \in \mathbb{N}$ and let $f \in C^{m}\left(\mathbb{R}^{N}\right)$. 
Suppose that all partial derivatives of $f$ of order $m$ are bounded, i.e.

$$
\left\|\frac{\partial^{m} f(\cdot, \cdot, \ldots, \cdot)}{\partial x_{1}^{\alpha_{1}} \ldots \partial x_{N}^{\alpha_{N}}}\right\|_{\infty}<\infty,
$$

for all $\alpha_{j} \in \mathbb{Z}^{+}, j=1, \ldots, N ; \sum_{j=1}^{N} \alpha_{j}=m$.

In this work we apply the general theory developed in [3] about $\theta_{r, n}^{[m]}$ to the operators $W_{r, n}^{[m]}$, so we can obtain computationaly specific results and show that the general theory has applications and it is a valid theory.

So for the very important in various branches of mathematics operators $W_{r, n}^{[m]}$ we prove the very essential properties of uniform approximation, $L_{p}$ approximation, global smoothness preservation and simultaneously approximation, Voronovskaya asymptotic expansions and complex simultaneous approximation.

\section{Auxilliary Essential Results}

We need the following:

Theorem 2.1. Let $r, N, m \in \mathbb{N}$, with $m>r ; \alpha_{j} \in \mathbb{Z}^{+}, j=1, \ldots, N:|\alpha|:=\sum_{j=1}^{N} \alpha_{j}=m, \xi_{n} \in(0,1]$, $n \in \mathbb{N}$. Then

$$
\begin{aligned}
\widetilde{u}_{\tilde{\xi}_{n}}(\alpha) & :=\frac{1}{\left(\sqrt{\pi \xi_{n}}\right)^{N}} \int_{\mathbb{R}^{N}}\left(\prod_{i=1}^{N}\left|s_{i}\right|^{\alpha_{i}}\right)\left(1+\frac{\|s\|_{2}}{\xi_{n}}\right)^{r} e^{-\frac{\left(\sum_{i=1}^{N} s_{i}^{2}\right)}{\xi_{n}}} d s_{1} \ldots d s_{N} \\
\leq & \left(\sqrt{\xi_{n}}\right)^{m-r}\left(\frac{2}{\sqrt{\pi}}\right)^{N}\left[(1+N)^{r}+2^{r}\left(\frac{\lfloor e(m+r) !\rfloor}{e}\right)^{N}\right] \\
& \leq\left(\frac{2}{\sqrt{\pi}}\right)^{N}\left[(1+N)^{r}+2^{r}\left(\frac{\lfloor e(m+r) !\rfloor}{e}\right)^{N}\right]<+\infty,
\end{aligned}
$$

are uniformly bounded.

Proof. We estimate

$$
\begin{gathered}
\widetilde{u}_{\xi_{n}}(\alpha):=\frac{1}{\left(\sqrt{\pi \xi_{n}}\right)^{N}} \int_{\mathbb{R}^{N}}\left(\prod_{i=1}^{N}\left|s_{i}\right|^{\alpha_{i}}\right)\left(1+\frac{\|s\|_{2}}{\xi_{n}}\right)^{r} e^{-\frac{\left(\sum_{i=1}^{N} s_{i}^{2}\right)}{\xi n}} d s_{1} \ldots d s_{N}= \\
\frac{2^{N}}{(\sqrt{\pi})^{N}\left(\sqrt{\xi_{n}}\right)^{N}} \int_{\mathbb{R}_{+}^{N}}\left(\prod_{i=1}^{N} s_{i}^{\alpha_{i}}\right)\left(1+\frac{\|s\|_{2}}{\xi_{n}}\right)^{r} e^{-\frac{\left(\sum_{i=1}^{N} s_{i}^{2}\right)}{\xi n}} d s_{1} \ldots d s_{N} \leq
\end{gathered}
$$




$$
\begin{aligned}
& \frac{2^{N}}{(\sqrt{\pi})^{N}\left(\sqrt{\xi_{n}}\right)^{N}} \int_{\mathbb{R}_{+}^{N}}\left(\prod_{i=1}^{N} s_{i}^{\alpha_{i}}\right)\left(1+\frac{\sum_{i=1}^{N} s_{i}}{\xi_{n}}\right)^{r} e^{-\frac{\left(\sum_{i=1}^{N} s_{i}^{2}\right)}{\xi_{n}}} d s_{1} \ldots d s_{N} \leq \\
& \frac{2^{N}\left(\sqrt{\xi_{n}}\right)^{m}}{(\sqrt{\pi})^{N}} \int_{\mathbb{R}_{+}^{N}}\left(\prod_{i=1}^{N}\left(\frac{s_{i}}{\sqrt{\xi_{n}}}\right)^{\alpha_{i}}\right)\left(\frac{1}{\sqrt{\xi_{n}}}+\frac{1}{\sqrt{\xi_{n}}} \sum_{i=1}^{N}\left(\frac{s_{i}}{\sqrt{\xi_{n}}}\right)\right)^{r} \\
& e^{-\sum_{i=1}^{N}\left(\frac{s_{i}}{\sqrt{\xi_{n}}}\right)^{2}} \frac{d s_{1}}{\sqrt{\xi_{n}}} \ldots \frac{d s_{N}}{\sqrt{\xi_{n}}}= \\
& \left(\frac{2}{\sqrt{\pi}}\right)^{N}\left(\sqrt{\xi_{n}}\right)^{m-r} \int_{\mathbb{R}_{+}^{N}}\left(\prod_{i=1}^{N} z_{i}^{\alpha_{i}}\right)\left(1+\sum_{i=1}^{N} z_{i}\right)^{r} e^{-\sum_{i=1}^{N} z_{i}^{2}} d z_{1} \ldots d z_{N}= \\
& \left(\sqrt{\xi_{n}}\right)^{m-r}\left(\frac{2}{\sqrt{\pi}}\right)^{N}\left[\int_{[0,1]^{N}}\left(\prod_{i=1}^{N} z_{i}^{\alpha_{i}}\right)\left(1+\sum_{i=1}^{N} z_{i}\right)^{r} e^{-\sum_{i=1}^{N} z_{i}^{2}} d z_{1} \ldots d z_{N}+\right. \\
& \left.\int_{\left(\mathbb{R}_{+}-[0,1]\right)^{N}}\left(\prod_{i=1}^{N} z_{i}^{\alpha_{i}}\right)\left(1+\sum_{i=1}^{N} z_{i}\right)^{r} e^{-\sum_{i=1}^{N} z_{i}^{2}} d z_{1} \ldots d z_{N}\right] \leq \\
& \left(\sqrt{\xi_{n}}\right)^{m-r}\left(\frac{2}{\sqrt{\pi}}\right)^{N}\left[\int_{[0,1]^{N}}\left(\prod_{i=1}^{N} z_{i}^{\alpha_{i}}\right)\left(1+\sum_{i=1}^{N} z_{i}\right)^{r} d z_{1} \ldots d z_{N}+\right. \\
& \left.2^{r} \int_{\left(\mathbb{R}_{+}-[0,1]\right)^{N}}\left(\prod_{i=1}^{N} z_{i}^{\alpha_{i}}\right)\left(\sum_{i=1}^{N} z_{i}\right)^{r} e^{-\sum_{i=1}^{N} z_{i}} d z_{1} \ldots d z_{N}\right] \leq \\
& \left(\sqrt{\xi_{n}}\right)^{m-r}\left(\frac{2}{\sqrt{\pi}}\right)^{N}\left[(1+N)^{r}+\right. \\
& \left.2^{r} \int_{\left(\mathbb{R}_{+}-[0,1]\right)^{N}}\left(\prod_{i=1}^{N} z_{i}^{\alpha_{i}}\right)\left(\prod_{i=1}^{N} z_{i}^{r}\right)\left(\prod_{i=1}^{N} e^{-z_{i}}\right) \prod_{i=1}^{N} d z_{i}\right]= \\
& \left(\sqrt{\xi_{n}}\right)^{m-r}\left(\frac{2}{\sqrt{\pi}}\right)^{N}\left[(1+N)^{r}+2^{r} \prod_{i=1}^{N} \int_{1}^{\infty} z_{i}^{\left(\left(\alpha_{i}+r\right)+1\right)-1} e^{-z_{i}} d z_{i}\right]=
\end{aligned}
$$

(by [7], p. 348)

$$
\left(\sqrt{\xi_{n}}\right)^{m-r}\left(\frac{2}{\sqrt{\pi}}\right)^{N}\left[(1+N)^{r}+2^{r} \prod_{i=1}^{N} \Gamma\left(\left(\alpha_{i}+r\right)+1,1\right)\right],
$$

where $\Gamma(\cdot, \cdot)$ is the upper incomplete gamma function.

We have proved that

$$
\widetilde{u}_{\xi_{n}}(\alpha) \leq\left(\sqrt{\xi_{n}}\right)^{m-r}\left(\frac{2}{\sqrt{\pi}}\right)^{N}\left[(1+N)^{r}+2^{r} \prod_{i=1}^{N} \Gamma\left(\left(\alpha_{i}+r\right)+1,1\right)\right] \leq
$$


COMPLETE APPROXIMATIONS BY MULTIVARIATE GENERALIZED GAUSS-WEIERSTRASS...

$$
\begin{gathered}
\left(\sqrt{\xi_{n}}\right)^{m-r}\left(\frac{2}{\sqrt{\pi}}\right)^{N}\left[(1+N)^{r}+2^{r}\left(\frac{\lfloor e(m+r) !\rfloor}{e}\right)^{N}\right] \leq \\
\left(\frac{2}{\sqrt{\pi}}\right)^{N}\left[(1+N)^{r}+2^{r}\left(\frac{\lfloor e(m+r) !\rfloor}{e}\right)^{N}\right]<+\infty,
\end{gathered}
$$

therefore $\widetilde{\mathcal{u}}_{\widetilde{\xi}_{n}}(\alpha)$ are uniformly bounded.

Above we used the formula

$$
\Gamma(s+1,1)=\frac{\lfloor e s !\rfloor}{e}, \quad s \in \mathbb{N} .
$$

Here $\alpha_{i}+r \in \mathbb{N}$, hence

$$
\Gamma\left(\left(\alpha_{i}+r\right)+1,1\right)=\frac{\left\lfloor e\left(\alpha_{i}+r\right) !\right\rfloor}{e} \leq \frac{\lfloor e(m+r) !\rfloor}{e}, \quad i=1, \ldots, N .
$$

The claim is proved.

We continue with

Theorem 2.2. Let $r, n, N \in \mathbb{N}, \xi_{n} \in(0,1]$. Then

$$
\begin{aligned}
\widetilde{\Phi}_{\xi_{n}}:= & \frac{1}{\left(\sqrt{\pi \xi_{n}}\right)^{N}} \int_{\mathbb{R}^{N}}\left(1+\frac{\|s\|_{2}}{\xi_{n}}\right)^{r} e^{-\frac{\left(\sum_{i=1}^{N} s_{i}^{2}\right)}{\xi_{n}}} d s_{1} \ldots d s_{N} \leq \\
& \frac{1}{\left(\sqrt{\xi_{n}}\right)^{r}}\left(\frac{2}{\sqrt{\pi}}\right)^{N}\left[(1+N)^{r}+2^{r}\left(\frac{\lfloor e r !\rfloor}{e}\right)^{N}\right] .
\end{aligned}
$$

Proof. We estimate

$$
\begin{gathered}
\widetilde{\Phi}_{\xi_{n}}=\frac{1}{\left(\sqrt{\pi \xi_{n}}\right)^{N}} \int_{\mathbb{R}^{N}}\left(1+\frac{\|s\|_{2}}{\xi_{n}}\right)^{r} e^{-\frac{\left(\sum_{i=1}^{N} s_{i}^{2}\right)}{\xi_{n}}} d s_{1} \ldots d s_{N}= \\
\frac{2^{N}}{(\sqrt{\pi})^{N}\left(\sqrt{\xi_{n}}\right)^{N}} \int_{\mathbb{R}_{+}^{N}}\left(1+\frac{\|s\|_{2}}{\xi_{n}}\right)^{r} e^{-\frac{\left(\sum_{i=1}^{N} s_{i}^{2}\right)}{\tilde{\xi}_{n}}} d s_{1} \ldots d s_{N} \leq \\
\left(\frac{2}{\sqrt{\pi}}\right)^{N} \int_{\mathbb{R}_{+}^{N}}\left(\frac{1}{\sqrt{\xi_{n}}}+\frac{\sum_{i=1}^{N} s_{i}}{\sqrt{\xi_{n}} \sqrt{\xi_{n}}}\right)^{r} e^{-\frac{\left(\sum_{i=1}^{N} s_{i}^{2}\right)}{\xi_{n}}} \frac{d s_{1}}{\sqrt{\xi_{n}} \ldots \frac{d s_{N}}{\sqrt{\xi_{n}}}}= \\
\left(\frac{2}{\sqrt{\pi}}\right)^{N} \frac{1}{\left(\sqrt{\xi_{n}}\right)^{r}} \int_{\mathbb{R}_{+}^{N}}\left(1+\sum_{i=1}^{N}\left(\frac{s_{i}}{\sqrt{\xi_{n}}}\right)\right)^{r} e^{-\sum_{i=1}^{N}\left(\frac{s_{i}}{\sqrt{\xi_{n}}}\right)^{2}} \frac{d s_{1}}{\sqrt{\xi_{n}} \ldots \frac{d s_{N}}{\sqrt{\xi_{n}}}}= \\
\left(\frac{2}{\sqrt{\pi}}\right)^{N} \frac{1}{\left(\sqrt{\xi_{n}}\right)^{r}} \int_{\mathbb{R}_{+}^{N}}\left(1+\sum_{i=1}^{N} z_{i}\right)^{r} e^{-\sum_{i=1}^{N} z_{i}^{2}} d z_{1} \ldots d z_{N}=
\end{gathered}
$$




$$
\begin{gathered}
\frac{1}{\left(\sqrt{\xi_{n}}\right)^{r}}\left(\frac{2}{\sqrt{\pi}}\right)^{N}\left[\int_{[0,1]^{N}}\left(1+\sum_{i=1}^{N} z_{i}\right)^{r} e^{-\sum_{i=1}^{N} z_{i}^{2}} d z_{1} \ldots d z_{N}+\right. \\
\left.\int_{\left(\mathbb{R}_{+}-[0,1]\right)^{N}}\left(1+\sum_{i=1}^{N} z_{i}\right)^{r} e^{-\sum_{i=1}^{N} z_{i}^{2}} d z_{1} \ldots d z_{N}\right] \leq \\
\frac{1}{\left(\sqrt{\xi_{n}}\right)^{r}}\left(\frac{2}{\sqrt{\pi}}\right)^{N}\left[\int_{[0,1]^{N}}\left(1+\sum_{i=1}^{N} z_{i}\right)^{r} d z_{1} \ldots d z_{N}+\right. \\
\frac{1}{\left(\sqrt{\xi_{n}}\right)^{r}}\left(\frac{2}{\sqrt{\pi})^{N}}\left[(1+N)^{r}+2^{r} \int_{\left(\mathbb{R}_{+}-[0,1]\right)^{N}}\left[\sum_{i=1}^{N} z_{i}\right)^{r} e^{-\sum_{i=1}^{N} z_{i}} d z_{1} \ldots d z_{N}\right] \leq\right. \\
\frac{1}{\left(\sqrt{\xi_{n}}\right)^{r}}\left(\frac{2}{\sqrt{\pi}}\right)^{N}\left[(1+N)^{r}+2^{r} \int_{\left(\mathbb{R}_{+}-[0,1]\right)^{N}}\left[\prod_{i=1}^{N} z_{i}^{r}\right)_{i=1}^{N} \prod_{i} e^{-z_{i}} \prod_{i=1}^{N} d z_{i}\right]= \\
\frac{1}{\left(\sqrt{\xi_{n}}\right)^{r}}\left(\frac{2}{\sqrt{\pi}}\right)^{N}\left[(1+N)^{r}+2^{r} \prod_{i=1}^{N} \int_{1}^{\infty} z_{i}^{r} e^{-z_{i}} d z_{i}\right]= \\
\frac{1}{\left(\sqrt{\xi_{n}}\right)^{r}}\left(\frac{2}{\sqrt{\pi}}\right)^{N}\left[(1+N)^{r}+2^{r}\left(\int_{1}^{\infty} z^{(r+1)-1} e^{-z} d z\right)^{N}\right]=
\end{gathered}
$$

(by [7], p. 348)

proving the claim.

$$
\begin{aligned}
& \frac{1}{\left(\sqrt{\xi_{n}}\right)^{r}}\left(\frac{2}{\sqrt{\pi}}\right)^{N}\left[(1+N)^{r}+2^{r} \Gamma^{N}(r+1,1)\right]= \\
& \frac{1}{\left(\sqrt{\xi_{n}}\right)^{r}}\left(\frac{2}{\sqrt{\pi}}\right)^{N}\left[(1+N)^{r}+2^{r}\left(\frac{\lfloor e r !\rfloor}{e}\right)^{N}\right],
\end{aligned}
$$

We give

Theorem 2.3. All as in Theorem 2.1 and $p>1$. Then

$$
\begin{gathered}
\widetilde{A}_{\xi_{n}}(\alpha):=\frac{1}{\left(\sqrt{\pi \xi_{n}}\right)^{N}} \int_{\mathbb{R}^{N}}\left(\left(\prod_{i=1}^{N}\left|s_{i}\right|^{\alpha_{i}}\right)\left(1+\frac{\|s\|_{2}}{\xi_{n}}\right)^{r}\right)^{p} e^{-\frac{\left(\sum_{i=1}^{N} s_{i}^{2}\right)}{\tilde{\xi}_{n}}} d s_{1} \ldots d s_{N} \leq \\
\left(\sqrt{\xi_{n}}\right)^{p(m-r)}\left(\frac{2}{\sqrt{\pi}}\right)^{N}\left[(1+N)^{r p}+2^{r p} \Gamma^{N}((m+r) p+1,1)\right] \leq \\
\left(\frac{2}{\sqrt{\pi}}\right)^{N}\left[(1+N)^{r p}+2^{r p} \Gamma^{N}((m+r) p+1,1)\right]<+\infty,
\end{gathered}
$$


are uniformly bounded, where $m>r$.

Proof. We estimate

$$
\begin{gathered}
\widetilde{A}_{\xi_{n}}(\alpha)=\frac{1}{\left(\sqrt{\pi \xi_{n}}\right)^{N}} \int_{\mathbb{R}^{N}}\left(\left(\prod_{i=1}^{N}\left|s_{i}\right|^{\alpha_{i}}\right)\left(1+\frac{\|s\|_{2}}{\xi_{n}}\right)^{r}\right)^{p} e^{-\frac{\left(\sum_{i=1}^{N} s_{i}^{2}\right)}{\xi_{n}}} d s_{1} \ldots d s_{N}= \\
\frac{2^{N}}{(\sqrt{\pi})^{N}\left(\sqrt{\xi_{n}}\right)^{N}} \int_{\mathbb{R}_{+}^{N}}\left(\prod_{i=1}^{N} s_{i}^{\alpha_{i} p}\right)\left(1+\frac{\|s\|_{2}}{\xi_{n}}\right)^{r p} e^{-\frac{\left(\sum_{i=1}^{N} s_{i}^{2}\right)}{\xi_{n}}} d s_{1} \ldots d s_{N} \leq \\
\left(\frac{2}{\sqrt{\pi}}\right)^{N}\left(\sqrt{\xi_{n}}\right)^{m p} \int_{\mathbb{R}_{+}^{N}}\left(\prod_{i=1}^{N}\left(\frac{s_{i}}{\sqrt{\xi_{n}}}\right)^{\alpha_{i} p}\right) \\
\left(\frac{\sum_{i=1}^{N} s_{i}}{\sqrt{\xi_{n}}}+\frac{\sum^{r}}{\sqrt{\xi_{n}} \sqrt{\xi_{n}}}\right)^{-\sum_{i=1}^{N}\left(\frac{s_{i}}{\sqrt{\xi_{n}}}\right)^{2}} \frac{d s_{1}}{\sqrt{\xi_{n}} \ldots \frac{d s_{N}}{\sqrt{\xi_{n}}}=}
\end{gathered}
$$

$\left(\right.$ set $\left.z_{i}=\frac{s_{i}}{\sqrt{\xi_{n}}}, i=1, \ldots, N\right)$

$$
\begin{aligned}
& \left(\frac{2}{\sqrt{\pi}}\right)^{N}\left(\sqrt{\xi_{n}}\right)^{p(m-r)} \int_{\mathbb{R}_{+}^{N}}\left(\prod_{i=1}^{N} z_{i}^{\alpha_{i} p}\right)\left(1+\sum_{i=1}^{N} z_{i}\right)^{r p} e^{-\sum_{i=1}^{N} z_{i}^{2}} d z_{1} \ldots d z_{N}= \\
& \left(\frac{2}{\sqrt{\pi}}\right)^{N}\left(\sqrt{\xi_{n}}\right)^{p(m-r)}\left[\int_{[0,1]^{N}}\left(\prod_{i=1}^{N} z_{i}^{\alpha_{i} p}\right)\left(1+\sum_{i=1}^{N} z_{i}\right)^{r p} e^{-\sum_{i=1}^{N} z_{i}^{2}} d z_{1} \ldots d z_{N}+\right. \\
& \left.\int_{\left(\mathbb{R}_{+}-[0,1]\right)^{N}}\left(\prod_{i=1}^{N} z_{i}^{\alpha_{i} p}\right)\left(1+\sum_{i=1}^{N} z_{i}\right)^{r p} e^{-\sum_{i=1}^{N} z_{i}^{2}} d z_{1} \ldots d z_{N}\right] \leq \\
& \left(\frac{2}{\sqrt{\pi}}\right)^{N}\left(\sqrt{\bar{\zeta}_{n}}\right)^{p(m-r)}\left[\int_{[0,1]^{N}}\left(\prod_{i=1}^{N} z_{i}^{\alpha_{i} p}\right)\left(1+\sum_{i=1}^{N} z_{i}\right)^{r p} d z_{1} \ldots d z_{N}+\right. \\
& \left.2^{r p} \int_{\left(\mathbb{R}_{+}-[0,1]\right)^{N}}\left(\prod_{i=1}^{N} z_{i}^{\alpha_{i} p}\right)\left(\sum_{i=1}^{N} z_{i}\right)^{r p} e^{-\sum_{i=1}^{N} z_{i}} d z_{1} \ldots d z_{N}\right] \leq \\
& \left(\frac{2}{\sqrt{\pi}}\right)^{N}\left(\sqrt{\bar{\zeta}_{n}}\right)^{p(m-r)} \\
& {\left[\frac{(1+N)^{r p}}{\prod_{i=1}^{N}\left(p \alpha_{i}+1\right)}+2^{r p} \int_{\left(\mathbb{R}_{+}-[0,1]\right)^{N}}\left(\prod_{i=1}^{N} z_{i}^{\alpha_{i} p}\right)\left(\prod_{i=1}^{N} z_{i}^{r p}\right) \prod_{i=1}^{N} e^{-z_{i}} \prod_{i=1}^{N} d z_{i}\right]}
\end{aligned}
$$


(by [7], p. 348)

$$
=\left(\sqrt{\xi}_{n}\right)^{p(m-r)}\left(\frac{2}{\sqrt{\pi}}\right)^{N}\left[\frac{(1+N)^{r p}}{\prod_{i=1}^{N}\left(p \alpha_{i}+1\right)}+2^{r p} \prod_{i=1}^{N} \int_{1}^{\infty} z_{i}^{\left(\alpha_{i}+r\right) p} e^{-z_{i}} d z_{i}\right]=
$$

$$
\left(\sqrt{\bar{\zeta}_{n}}\right)^{p(m-r)}\left(\frac{2}{\sqrt{\pi}}\right)^{N}\left[\frac{(1+N)^{r p}}{\prod_{i=1}^{N}\left(p \alpha_{i}+1\right)}+2^{r p} \prod_{i=1}^{N} \Gamma\left(\left(\alpha_{i}+r\right) p+1,1\right)\right] \leq
$$

(by [7], p. 909)

$$
\left(\sqrt{\bar{\zeta}_{n}}\right)^{p(m-r)}\left(\frac{2}{\sqrt{\pi}}\right)^{N}\left[(1+N)^{r p}+2^{r p} \Gamma^{N}((m+r) p+1,1)\right] .
$$

We have proved that

$$
\begin{aligned}
\widetilde{A}_{\xi_{n}}(\alpha) \leq & \left(\sqrt{\xi_{n}}\right)^{p(m-r)}\left(\frac{2}{\sqrt{\pi}}\right)^{N}\left[(1+N)^{r p}+2^{r p} \Gamma^{N}((m+r) p+1,1)\right] \leq \\
& \left(\frac{2}{\sqrt{\pi}}\right)^{N}\left[(1+N)^{r p}+2^{r p} \Gamma^{N}((m+r) p+1,1)\right]<+\infty,
\end{aligned}
$$

are uniformly bounded, where $m>r$.

Above we used ([7], p. 909) the formula

$$
\Gamma(\alpha, x y)=y^{\alpha} e^{-x y} \int_{0}^{\infty} e^{-t y}(t+x)^{\alpha-1} d t
$$

where $\operatorname{Rey}>0, x>0, \operatorname{Re} \alpha>1$.

We notice that $\left(x=y=1, \alpha_{i} \leq m\right)$

$$
\begin{gathered}
\Gamma\left(\left(\alpha_{i}+r\right) p+1,1\right)=e^{-1} \int_{0}^{\infty} e^{-t}(t+1)^{\left(\alpha_{i}+r\right) p} d t \leq \\
e^{-1} \int_{0}^{\infty} e^{-t}(t+1)^{(m+r) p} d t=\Gamma((m+r) p+1,1) .
\end{gathered}
$$

That is

$$
\Gamma\left(\left(\alpha_{i}+r\right) p+1,1\right) \leq \Gamma((m+r) p+1,1)
$$

for all $i=1, \ldots, N$.

The theorem is proved.

We continue with

Theorem 2.4. Let $r, n, N \in \mathbb{N}, \xi_{n} \in(0,1], p>1$. Then

$$
B_{\xi_{n}}:=\frac{1}{\left(\sqrt{\pi \xi_{n}}\right)^{N}} \int_{\mathbb{R}^{N}}\left(1+\frac{\|s\|_{2}}{\xi_{n}}\right)^{r p} e^{-\frac{\left(\sum_{i=1}^{N} s_{i}^{2}\right)}{\xi_{n}}} d s_{1} \ldots d s_{N} \leq
$$


COMPLETE APPROXIMATIONS BY MULTIVARIATE GENERALIZED GAUSS-WEIERSTRASS...

$$
\frac{1}{\left(\sqrt{\xi_{n}}\right)^{p r}}\left(\frac{2}{\sqrt{\pi}}\right)^{N}\left[(1+N)^{p r}+2^{p r} \Gamma^{N}(r p+1,1)\right] .
$$

Proof. We estimate

$$
\begin{aligned}
& B_{\xi_{n}}=\frac{1}{\left(\sqrt{\pi \xi_{n}}\right)^{N}} \int_{\mathbb{R}^{N}}\left(1+\frac{\|s\|_{2}}{\xi_{n}}\right)^{p r} e^{-\frac{\left(\sum_{i=1}^{N} s_{i}^{2}\right)}{\xi_{n}}} d s_{1} \ldots d s_{N}= \\
& \frac{2^{N}}{(\sqrt{\pi})^{N}\left(\sqrt{\xi_{n}}\right)^{N}} \int_{\mathbb{R}_{+}^{N}}\left(1+\frac{\|s\|_{2}}{\xi_{n}}\right)^{p r} e^{-\frac{\left(\sum_{i=1}^{N} s_{i}^{2}\right)}{\xi_{n}}} d s_{1} \ldots d s_{N} \leq
\end{aligned}
$$

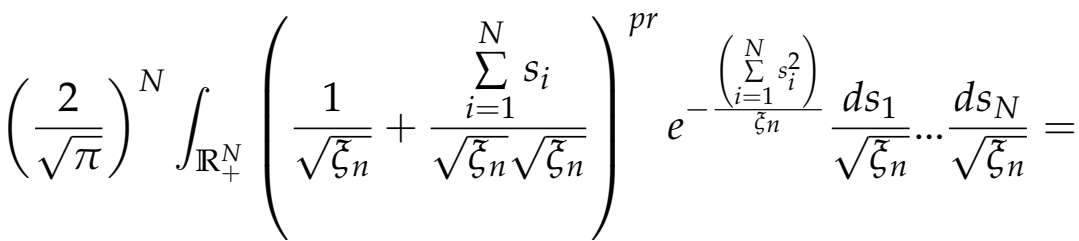

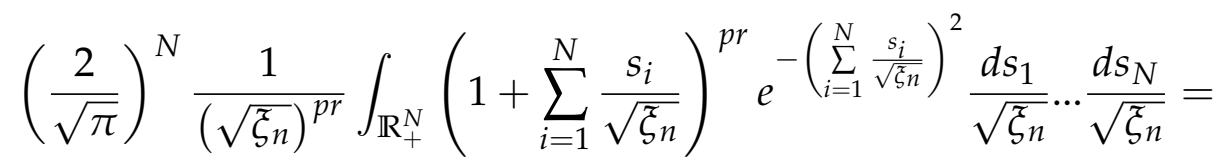

$$
\begin{aligned}
& \left(\frac{2}{\sqrt{\pi}}\right)^{N} \frac{1}{\left(\sqrt{\xi_{n}}\right)^{p r}} \int_{\mathbb{R}_{+}^{N}}\left(1+\sum_{i=1}^{N} z_{i}\right)^{p r} e^{-\sum_{i=1}^{N} z_{i}^{2}} d z_{1} \ldots d z_{N}= \\
& \frac{1}{\left(\sqrt{\xi_{n}}\right)^{p r}}\left(\frac{2}{\sqrt{\pi}}\right)^{N}\left[\int_{[0,1]^{N}}\left(1+\sum_{i=1}^{N} z_{i}\right)^{p r} e^{-\sum_{i=1}^{N} z_{i}^{2}} d z_{1} \ldots d z_{N}+\right. \\
& \left.\int_{\left(\mathbb{R}_{+}-[0,1]\right)^{N}}\left(1+\sum_{i=1}^{N} z_{i}\right)^{p r} e^{-\sum_{i=1}^{N} z_{i}^{2}} d z_{1} \ldots d z_{N}\right] \leq \\
& \frac{1}{\left(\sqrt{\xi_{n}}\right)^{p r}}\left(\frac{2}{\sqrt{\pi}}\right)^{N}\left[(1+N)^{p r}+2^{p r} \int_{\left(\mathbb{R}_{+}-[0,1]\right)^{N}}\left(\sum_{i=1}^{N} z_{i}\right)^{p r} e^{-\sum_{i=1}^{N} z_{i}} d z_{1} \ldots d z_{N}\right] \leq \\
& \frac{1}{\left(\sqrt{\xi_{n}}\right)^{p r}}\left(\frac{2}{\sqrt{\pi}}\right)^{N}\left[(1+N)^{p r}+2^{p r} \int_{\left(\mathbb{R}_{+}-[0,1]\right)^{N}}\left(\prod_{i=1}^{N} z_{i}^{p r}\right) \prod_{i=1}^{N} e^{-z_{i}} \prod_{i=1}^{N} d z_{i}\right]= \\
& \frac{1}{\left(\sqrt{\xi_{n}}\right)^{p r}}\left(\frac{2}{\sqrt{\pi}}\right)^{N}\left[(1+N)^{p r}+2^{p r} \prod_{i=1}^{N} \int_{1}^{\infty} z_{i}^{p r} e^{-z_{i}} d z_{i}\right]=
\end{aligned}
$$

(by [7], p. 348)

$$
\frac{1}{\left(\sqrt{\xi_{n}}\right)^{p r}}\left(\frac{2}{\sqrt{\pi}}\right)^{N}\left[(1+N)^{p r}+2^{p r} \Gamma^{N}(r p+1,1)\right]
$$

proving the claim. 
We finish this section with

Theorem 2.5. Let $n, N \in \mathbb{N}, \xi_{n} \in(0,1], \alpha_{j} \in \mathbb{Z}^{+}, j=1, \ldots, N:|\alpha|:=\sum_{j=1}^{N} \alpha_{j}=m \in \mathbb{N}$. Then

$$
\begin{aligned}
& D_{\xi_{n}}(\alpha):=
\end{aligned}
$$

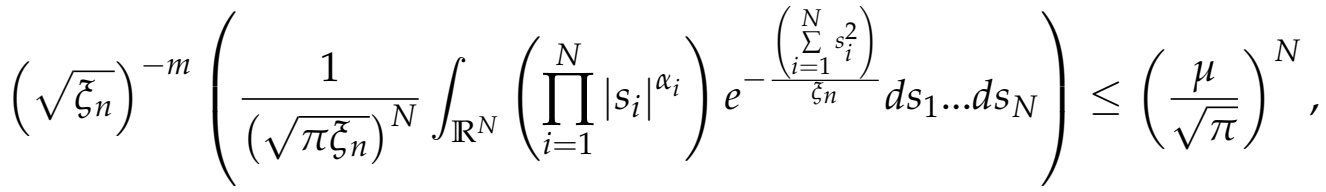

where

$$
\mu:=\max \left\{\sqrt{\pi}, \Gamma\left(\frac{m+1}{2}\right)\right\}
$$

where $\Gamma$ is the gamma function.

Proof. We have that

$$
\begin{gathered}
D_{\xi_{n}}(\alpha) \leq\left(\sqrt{\xi_{n}}\right)^{-m}\left(\frac{1}{\left(\sqrt{\pi \xi_{n}}\right)^{N}} \int_{\mathbb{R}^{N}}\left(\prod_{i=1}^{N}\left|s_{i}\right|^{\alpha_{i}}\right) e^{-\frac{\left(\sum_{i=1}^{N} s_{i}^{2}\right)}{\xi_{n}}} d s_{1} \ldots d s_{N}\right)= \\
\left(\sqrt{\xi_{n}}\right)^{-m}\left(\frac{2^{N}}{\left(\sqrt{\pi \xi_{n}}\right)^{N}} \int_{\mathbb{R}_{+}^{N}}\left(\prod_{i=1}^{N} s_{i}^{\alpha_{i}}\right) e^{-\frac{\left(\sum_{i=1}^{N} s_{i}^{2}\right)}{\xi_{n}}} d s_{1} \ldots d s_{N}\right)= \\
\left(\sqrt{\xi_{n}}\right)^{-m}\left(\left(\frac{2}{\sqrt{\pi}}\right)^{N}\left(\sqrt{\xi_{n}}\right)^{m} \int_{\mathbb{R}_{+}^{N}}\left(\prod_{i=1}^{N}\left(\frac{s_{i}}{\sqrt{\xi_{n}}}\right)^{\alpha_{i}}\right) e^{-\sum_{i=1}^{N}\left(\frac{s_{i}}{\sqrt{\xi_{n}}}\right)^{2}} \frac{d s_{1}}{\sqrt{\xi_{n}}} \ldots \frac{d s_{N}}{\sqrt{\xi_{n}}}\right)= \\
\left(\frac{2}{\sqrt{\pi}}\right)^{N} \int_{\mathbb{R}_{+}^{N}}\left(\prod_{i=1}^{N} z_{i}^{\alpha_{i}}\right) e^{-\sum_{i=1}^{N} z_{i}^{2}} d z_{1} \ldots d z_{n}= \\
\left(\frac{2}{\sqrt{\pi}}\right)^{N} \prod_{i=1}^{N} \int_{0}^{\infty} z_{i}^{\alpha_{i}} e^{-z_{i}^{2} d z_{i}=}
\end{gathered}
$$

(by [7], p. 349)

$$
\left(\left(\frac{2}{\sqrt{\pi}}\right)^{N} \prod_{i=1}^{N}\left(\frac{\Gamma\left(\frac{\alpha_{i}+1}{2}\right)}{2}\right)\right)=\frac{1}{(\sqrt{\pi})^{N}}\left(\prod_{i=1}^{N} \Gamma\left(\frac{\alpha_{i}+1}{2}\right)\right) \leq(*) .
$$

Some important values of $\Gamma\left(\frac{\alpha_{i}+1}{2}\right)$ follow:

if $\alpha_{i}=0$, then $\Gamma\left(\frac{1}{2}\right)=\sqrt{\pi} \approx 1.77245 \ldots$,

if $\alpha_{i}=1$, then $\Gamma(1)=1$,

if $\alpha_{i}=2$, then $\Gamma\left(\frac{3}{2}\right)=\frac{\sqrt{\pi}}{2} \approx 0.886226 \ldots$,

if $\alpha_{i}=3$, then $\Gamma(2)=1$, 
if $\alpha_{i}=4$, then $\Gamma\left(\frac{5}{2}\right)=\frac{3 \sqrt{\pi}}{4} \approx 1.32934$,

if $\alpha_{i}=5$, then $\Gamma(3)=2$.

The function $\Gamma$ has minimum at $x_{\min }=1.46 \ldots$, with $\Gamma\left(x_{\min }\right)=0.885 \ldots$, and $\Gamma(x)$ is increasing for $x \geq x_{\min }$. To have $\frac{\alpha_{i}+1}{2}>1.46 \ldots$, we need $\alpha_{i}>1.92 \ldots$ Thus when $\alpha_{i} \geq 2$, then $\Gamma\left(\frac{\alpha_{i}+1}{2}\right)$ increases, and $\Gamma\left(\frac{\alpha_{i}+1}{2}\right) \leq \Gamma\left(\frac{m+1}{2}\right)$, when $m \geq 2$.

Hence it holds $\Gamma\left(\frac{\alpha_{i}+1}{2}\right) \leq \mu$, for $i=1, \ldots, N$, and

$$
(*) \leq\left(\frac{\mu}{\sqrt{\pi}}\right)^{N}
$$

proving the claim.

\section{Main Results for $W_{r, n}^{[m]}$}

3.1. Uniform Approximation. We start with an application to $W_{r, n}^{[m]}$ of the following theorem.

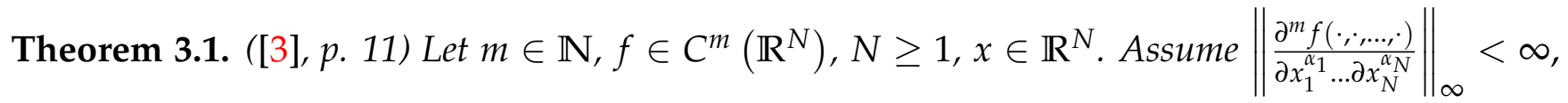
for all $\alpha_{j} \in \mathbb{Z}^{+}, j=1, \ldots, N:|\alpha|:=\sum_{i=1}^{N} \alpha_{i}=m$. Let $\mu_{\xi_{n}}$ be a Borel probability measure on $\mathbb{R}^{N}$, for $\xi_{n}>0,\left(\xi_{n}\right)_{n \in \mathbb{N}}$ bounded sequence.

Suppose that for all $\alpha:=\left(\alpha_{1}, \ldots, \alpha_{N}\right), \alpha_{i} \in \mathbb{Z}^{+}, i=1, \ldots, N,|\alpha|:=\sum_{i=1}^{N} \alpha_{i}=m$ we have that

$$
u_{\xi_{n}}(\alpha):=\int_{\mathbb{R}^{N}}\left(\prod_{i=1}^{N}\left|s_{i}\right|^{\alpha_{i}}\right)\left(1+\frac{\|s\|_{2}}{\xi_{n}}\right)^{r} d \mu_{\xi_{n}}(s)<\infty .
$$

For $\widetilde{j}=1, \ldots, m$, and $\alpha:=\left(\alpha_{1}, \ldots, \alpha_{N}\right), \alpha_{i} \in \mathbb{Z}^{+}, i=1, \ldots, N,|\alpha|:=\sum_{j=1}^{N} \alpha_{j}=\widetilde{j}$, call

$$
c_{\alpha, n}:=c_{\alpha, n, \tilde{j}}:=\int_{\mathbb{R}^{N}} \prod_{i=1}^{N} s_{i}^{\alpha_{i}} d \mu_{\xi_{n}}\left(s_{1}, \ldots, s_{N}\right) .
$$

Then

i)

$$
\begin{aligned}
& E_{r, n}^{[m]}(x):=\left|\theta_{r, n}^{[m]}(f ; x)-f(x)-\sum_{\widetilde{j}=1}^{m} \delta_{\widetilde{j}, r}^{[m]}\left(\sum_{\substack{\alpha_{1}, \ldots, \alpha_{N} \geq 0 ; \\
|\alpha|=\tilde{j}}} \frac{c_{\alpha, n, \tilde{j}^{j}} f_{\alpha}(x)}{\prod_{i=1}^{N} \alpha_{i} !}\right)\right| \\
& \leq \sum_{\substack{\alpha_{1}, \ldots, \alpha_{N} \geq 0 ; \\
|\alpha|=m}} \frac{\left(\omega_{r}\left(f_{\alpha}, \xi_{n}\right)\right)}{\left(\prod_{i=1}^{N} \alpha_{i}\right)}\left(\int_{\mathbb{R}^{N}}\left(\prod_{i=1}^{N}\left|s_{i}\right|^{\alpha_{i}}\right)\left(1+\frac{\|s\|_{2}}{\xi_{n}}\right)^{r} d \mu_{\xi_{n}}(s)\right) .
\end{aligned}
$$


$\forall x \in \mathbb{R}^{N}$.

ii)

$$
\left\|E_{r, n}^{[m]}\right\|_{\infty} \leq \text { R.H.S.(55). }
$$

Given that $\xi_{n} \rightarrow 0$, as $n \rightarrow \infty$, and $u_{\xi_{n}}$ is uniformly bounded, then we derive that $\left\|E_{r, n}^{[m]}\right\| \rightarrow 0$ with rates.

iii) It holds also that

$$
\left\|\theta_{r, n}^{[m]}(f)-f\right\|_{\infty} \leq \sum_{\tilde{j}=1}^{m} \delta_{\widetilde{j}, r}^{[m]}\left(\sum_{\substack{\alpha_{1}, \ldots, \alpha_{N} \geq 0 ; \\|\alpha|=\tilde{j}}} \frac{\left|c_{\alpha, n, \tilde{j}}\right|\left\|f_{\alpha}\right\|_{\infty}}{\prod_{i=1}^{N} \alpha_{i} !}\right)+\text { R.H.S.(55), }
$$

given that $\left\|f_{\alpha}\right\|_{\infty}<\infty$, for all $\alpha:|\alpha|=\widetilde{j}, \widetilde{j}=1, \ldots, m$. Furthermore, as $\xi_{n} \rightarrow 0$ when $n \rightarrow \infty$, assuming that $c_{\alpha, n, \tilde{j}} \rightarrow 0$, while $u_{\xi_{n}}$ is uniformly bounded, we conclude that

$$
\left\|\theta_{r, n}^{[m]}(f)-f\right\|_{\infty} \rightarrow 0
$$

with rates.

A uniform approximation result for $W_{r, n}^{[m]}$ follows:

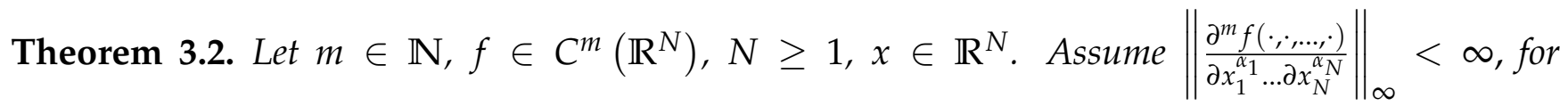
all $\alpha_{j} \in \mathbb{Z}^{+}, j=1, \ldots, N:|\alpha|:=\sum_{j=1}^{N} \alpha_{j}=m$. Here $\xi_{n} \in(0,1], n \in \mathbb{N}, m>r \in \mathbb{N}$. For all $\alpha:=\left(\alpha_{1}, \ldots, \alpha_{N}\right), \alpha_{i} \in \mathbb{Z}^{+}, i=1, \ldots, N,|\alpha|:=\sum_{i=1}^{N} \alpha_{i}=m$, we denote

$$
\widetilde{u}_{\xi_{n}}(\alpha):=\frac{1}{\left(\sqrt{\pi \xi_{n}}\right)^{N}} \int_{\mathbb{R}^{N}}\left(\prod_{i=1}^{N}\left|s_{i}\right|^{\alpha_{i}}\right)\left(1+\frac{\|s\|_{2}}{\xi_{n}}\right)^{r} e^{-\frac{\left(\sum_{i=1}^{N} s_{i}^{2}\right)}{\xi_{n}}} d s_{1} \ldots d s_{N} .
$$

For $\widetilde{j}=1, \ldots, m$, and $\alpha:=\left(\alpha_{1}, \ldots, \alpha_{N}\right), \alpha_{i} \in \mathbb{Z}^{+}, i=1, \ldots, N,|\alpha|:=\sum_{i=1}^{N} \alpha_{i}=\widetilde{j}$, call

$$
\widetilde{c}_{\alpha, n}:=\widetilde{c}_{\alpha, n, \tilde{j}}:=\frac{1}{\left(\sqrt{\pi \widetilde{\zeta}_{n_{n}}}\right)^{N}} \int_{\mathbb{R}^{N}} \prod_{i=1}^{N} s_{i}^{\alpha_{i}} e^{-\frac{\left(\sum_{i=1}^{N} s_{i}^{2}\right)}{\xi_{n}}} d s_{1} \ldots d s_{N} .
$$

Then

i)

$$
\widetilde{E}_{r, n}^{[m]}(x):=\left|W_{r, n}^{[m]}(f ; x)-f(x)-\sum_{\tilde{j}=1}^{m} \delta_{\widetilde{j}, r}^{[m]}\left(\sum_{\substack{\alpha_{1}, \ldots, \alpha_{N} \geq 0 ; \\|\alpha|=\tilde{j}}} \frac{\widetilde{c}_{\alpha, n, \widetilde{j}^{\prime}} f_{\alpha}(x)}{\prod_{i=1}^{N} \alpha_{i} !}\right)\right|
$$




$$
\leq \sum_{\substack{\alpha_{1}, \ldots, \alpha_{N} \geq 0 ; \\|\alpha|=m}} \frac{\left(\omega_{r}\left(f_{\alpha}, \xi_{n}\right)\right)}{\left(\prod_{i=1}^{N} \alpha_{i} !\right)} \widetilde{u}_{\xi_{n}}(\alpha)
$$

$\forall x \in \mathbb{R}^{N}$.

ii)

$$
\left\|\widetilde{E}_{r, n}^{[m]}\right\|_{\infty} \leq \text { R.H.S.(61). }
$$

Given that $\xi_{n} \rightarrow 0$, as $n \rightarrow+\infty$, we have that $\widetilde{u}_{\xi_{n}}(\alpha) \rightarrow 0$ and are uniformly bounded, and then we derive that $\left\|\widetilde{E}_{r, n}^{[m]}\right\|_{\infty} \rightarrow 0$ with rates.

iii) It holds also that

$$
\left\|W_{r, n}^{[m]}(f)-f\right\|_{\infty} \leq \sum_{\widetilde{j}=1}^{m}\left|\delta_{\widetilde{j}, r}^{[m]}\right|\left(\sum_{\substack{\alpha_{1}, \ldots, \alpha_{N} \geq 0 ; \\|\alpha|=\tilde{j}}} \frac{\left|\widetilde{c}_{\alpha, n, \tilde{j}}\right|\left\|f_{\alpha}\right\|_{\infty}}{\prod_{i=1}^{N} \alpha_{i} !}\right)+\text { R.H.S.(61), }
$$

given that $\left\|f_{\alpha}\right\|_{\infty}<+\infty$, for all $\alpha:|\alpha|=\widetilde{j}, \widetilde{j}=1, \ldots, m$. Furthermore, as $\xi_{n} \rightarrow 0$ when $n \rightarrow+\infty$, we have that $\widetilde{c}_{\alpha, n, \tilde{j}} \rightarrow 0$ and $\widetilde{u}_{\xi_{n}}(\alpha) \rightarrow 0$, and both are uniformly bounded, and we conclude that

$$
\left\|W_{r, n}^{[m]}(f)-f\right\|_{\infty} \rightarrow 0
$$

with rates.

Proof. Mainly by applying Theorem 3.1. By Theorem 2.1 we get that $\widetilde{u}_{\xi_{n}}(\alpha) \rightarrow 0$ and $\widetilde{u}_{\xi_{n}}(\alpha)$ are uniformly bounded. By Theorem 2.5 we get that

$$
\begin{aligned}
\left|\widetilde{c}_{\alpha, n}\right|=\left|\widetilde{c}_{\alpha, n, \tilde{j}}\right| & \leq \frac{1}{\left(\sqrt{\pi \xi_{n}}\right)^{N}} \int_{\mathbb{R}^{N}}\left(\prod_{i=1}^{N}\left|s_{i}\right|^{\alpha_{i}}\right) e^{-\frac{\left(\sum_{i=1}^{N} s_{i}^{2}\right)}{\xi n}} d s_{1} \ldots d s_{N} \\
& \leq\left(\frac{\mu}{\sqrt{\pi}}\right)^{N}\left(\sqrt{\widetilde{\zeta}_{n}}\right)^{m} \leq\left(\frac{\mu}{\sqrt{\pi}}\right)^{N} .
\end{aligned}
$$

That is $\widetilde{c}_{\alpha, n, \widetilde{j}} \rightarrow 0$ and are uniformly bounded. The proof is complete.

We mention

Theorem 3.3. ([3], p. 14) Let $f \in C_{B}\left(\mathbb{R}^{N}\right), N \geq 1, \xi_{n} \in(0,1]$. Then

$$
\left\|\theta_{r, n}^{[0]} f-f\right\|_{\infty} \leq\left(\int_{\mathbb{R}^{N}}\left(1+\frac{\|s\|_{2}}{\xi_{n}}\right)^{r} d \mu_{\xi_{n}}(s)\right) \omega_{r}\left(f, \xi_{n}\right),
$$

under the assumption

$$
\Phi_{\xi_{n}}:=\int_{\mathbb{R}^{N}}\left(1+\frac{\|s\|_{2}}{\xi_{n}}\right)^{r} d \mu_{\xi_{n}}(s)<\infty
$$


In case of $f$ being uniformly, continuous and as $n \rightarrow \infty$ and $\xi_{n} \rightarrow 0$, given that $\Phi_{\xi_{n}}$ are uniformly bounded, we derive

with rates.

$$
\left\|\theta_{r, n}^{[0]} f-f\right\|_{\infty} \rightarrow 0
$$

We give

Theorem 3.4. Let $f \in C_{B}\left(\mathbb{R}^{N}\right), N \geq 1, \xi_{n} \in(0,1]$. Then

$$
\left\|W_{r, n}^{[0]} f-f\right\|_{\infty} \leq\left(\frac{2}{\sqrt{\pi}}\right)^{N}\left[(1+N)^{r}+2^{r}\left(\frac{\lfloor e r !\rfloor}{e}\right)^{N}\right] \frac{\omega_{r}\left(f, \xi_{n}\right)}{\left(\sqrt{\xi_{n}}\right)^{r}}
$$

where $r, n \in \mathbb{N}$.

2) Let $r \in \mathbb{N}-\{1\}, 0<\alpha \leq 1$, and consider $f \in C_{B}\left(\mathbb{R}^{N}\right)$ such that $\omega_{r}(f, t) \leq K t^{r-1+\alpha}, \forall t>0$, where $K>0$ is independent of $t$.

In this case

$$
\frac{\omega_{r}\left(f, \xi_{n}\right)}{\left(\sqrt{\xi_{n}}\right)^{r}} \leq K \xi_{n}^{\left(\frac{r}{2}+\alpha-1\right)}
$$

and as $n \rightarrow \infty$ and $\xi_{n} \rightarrow 0$, we derive

$$
\left\|W_{r, n}^{[0]} f-f\right\|_{\infty} \rightarrow 0
$$

Proof. By Theorems 2.2 and 3.3. For (69), since $r \geq 2$, we get that $\frac{r}{2}+\alpha-1>0$.

3.2. $L_{p}$ Approximation for $W_{r, n}^{[m]}$. We need

Definition 3.1. ([4], [8]) We call

$$
\begin{gathered}
\Delta_{u}^{r} f(x):=\Delta_{u_{1}, u_{2}, \ldots, u_{N}}^{r} f\left(x_{1}, \ldots, x_{N}\right):= \\
\sum_{j=0}^{r}(-1)^{r-j}\left(\begin{array}{c}
r \\
j
\end{array}\right) f\left(x_{1}+j u_{1}, x_{2}+j u_{2}, \ldots, x_{N}+j u_{N}\right) .
\end{gathered}
$$

Let $p \geq 1$, the modulus of smoothness of order $r$ is given by

$$
\omega_{r}(f ; h)_{p}:=\sup _{\|u\|_{2} \leq h}\left\|\Delta_{u}^{r}(f)\right\|_{p}
$$

$h>0$.

We will apply

Theorem 3.5. ([3], p. 24) Let $f \in C^{m}\left(\mathbb{R}^{N}\right), m \in \mathbb{N}, N \geq 1$, with $f_{\alpha} \in L_{p}\left(\mathbb{R}^{N}\right),|\alpha|=m, x \in \mathbb{R}^{N}$. Let $p, q>1: \frac{1}{p}+\frac{1}{q}=1$. Here, $\mu_{\xi_{n}}$ is a Borel probability measure on $\mathbb{R}^{N}$ for $\xi_{n}>0,\left(\xi_{n}\right)_{n \in \mathbb{N}}$ bounded sequence. Assume for all $\alpha:=\left(\alpha_{1}, \ldots, \alpha_{N}\right), \alpha_{i} \in \mathbb{Z}^{+}, i=1, \ldots, N,|\alpha|:=\sum_{i=1}^{N} \alpha_{i}=m$ that we have

$$
\int_{\mathbb{R}^{N}}\left(\left(\prod_{i=1}^{N}\left|s_{i}\right|^{\alpha_{i}}\right)\left(1+\frac{\|s\|_{2}}{\xi_{n}}\right)^{r}\right)^{p} d \mu_{\xi_{n}}(s)<\infty .
$$


For $\widetilde{j}=1, \ldots, m$, and $\alpha:=\left(\alpha_{1}, \ldots, \alpha_{N}\right), \alpha_{i} \in \mathbb{Z}^{+}, i=1, \ldots, N,|\alpha|:=\sum_{j=1}^{N} \alpha_{j}=\widetilde{j}$, call

$$
c_{\alpha, n, \tilde{j}}:=\int_{\mathbb{R}^{N}} \prod_{i=1}^{N} s_{i}^{\alpha_{i}} d \mu_{\xi_{n}}(s) .
$$

Then

$$
\begin{gathered}
\left\|E_{r, n}^{[m]}\right\|_{p}=\left\|\theta_{r, n}^{[m]}(f ; x)-f(x)-\sum_{\tilde{j}=1}^{m} \delta_{\tilde{j}, r}^{[m]}\left(\sum_{|\alpha|=\widetilde{j}} \frac{c_{\alpha, n, \tilde{j}} f_{\alpha}(x)}{\left(\prod_{i=1}^{N} \alpha_{i} !\right)}\right)\right\|_{p, x} \\
\leq\left(\frac{m}{(q(m-1)+1)^{\frac{1}{q}}}\right)\left(\sum_{|\alpha|=m} \frac{1}{\prod_{i=1}^{N} \alpha_{i} !}\right) \\
{\left[\int_{\mathbb{R}^{N}}\left[\left(\prod_{i=1}^{N}\left|s_{i}\right|^{\alpha_{i}}\right)\left(1+\frac{\|s\|_{2}}{\xi_{n}}\right)^{r}\right]^{p} d \mu_{\xi_{n}}(s)\right]^{\frac{1}{p}} \omega_{r}\left(f_{\alpha}, \xi_{n}\right)_{p} .}
\end{gathered}
$$

As $n \rightarrow \infty$ and $\xi_{n} \rightarrow 0$, by (75) we obtain that $\left\|E_{r, n}^{[m]}\right\| \rightarrow 0$ with rates.

One also finds by (75) that

$$
\left\|\theta_{r, n}^{[m]}(f ; x)-f(x)\right\|_{p, x} \leq \sum_{\tilde{j}=1}^{m}\left|\delta_{\widetilde{j}, r}^{[m]}\right|\left(\sum_{|\alpha|=\tilde{j}} \frac{\left|c_{\alpha, n, \tilde{j}}\right|}{\prod_{i=1}^{N} \alpha_{i} !}\left\|f_{\alpha}\right\|_{p}\right)+\text { R.H.S.(75), }
$$

given that $\left\|f_{\alpha}\right\|_{p}<\infty,|\alpha|=\widetilde{j}, \widetilde{j}=1, \ldots, m$.

Assuming that $c_{\alpha, n, \tilde{j}} \rightarrow 0, \xi_{n} \rightarrow 0$, as $n \rightarrow \infty$, we get $\left\|\theta_{r, n}^{[m]}(f)-f\right\|_{p} \rightarrow 0$, that is $\theta_{r, n}^{[m]} \rightarrow I$ the unit operator, in $L_{p}$ norm, with rates.

We present

Theorem 3.6. Let $f \in C^{m}\left(\mathbb{R}^{N}\right), m \in \mathbb{N}, m>r, N \geq 1$, with $f_{\alpha} \in L_{p}\left(\mathbb{R}^{N}\right),|\alpha|=m, x \in \mathbb{R}^{N}$. Let $p, q>1: \frac{1}{p}+\frac{1}{q}=1, \xi_{n} \in(0,1]$. For $\widetilde{j}=1, \ldots, m$, and $\alpha:=\left(\alpha_{1}, \ldots, \alpha_{N}\right), \alpha_{i} \in \mathbb{Z}^{+}, i=1, \ldots, N$, $|\alpha|:=\sum_{i=1}^{N} \alpha_{i}=\widetilde{j}$, call

$$
\widetilde{c}_{\alpha, n, \tilde{j}}=\frac{1}{\left(\sqrt{\pi \xi_{n}}\right)^{N}} \int_{\mathbb{R}^{N}} \prod_{i=1}^{N} s_{i}^{\alpha_{i}} e^{-\frac{\left(\sum_{i=1}^{N} s_{i}^{2}\right)}{\tilde{\zeta}^{2} n}} d s_{1} \ldots d s_{N} .
$$


Then

$$
\begin{aligned}
&\left\|\widetilde{E}_{r, n}^{[m]}\right\|_{p}=\left\|W_{r, n}^{[m]}(f ; x)-f(x)-\sum_{\widetilde{j}=1}^{m} \delta_{\widetilde{j}, r}^{[m]}\left(\sum_{|\alpha|=\tilde{j}} \frac{\widetilde{c}_{\alpha, n, \tilde{j}} f_{\alpha}(x)}{\left(\prod_{i=1}^{N} \alpha_{i} !\right)}\right)\right\|_{p, x} \\
& \leq\left(\frac{2}{\sqrt{\pi}}\right)^{\frac{N}{p}}\left(\frac{m}{(q(m-1)+1)^{\frac{1}{q}}}\right)\left[(1+N)^{r p}+2^{r p} \Gamma^{N}((m+r) p+1,1)\right]^{\frac{1}{p}} \\
&\left(\sum_{|\alpha|=m} \frac{1}{\prod_{i=1}^{N} \alpha_{i} !}\right)\left(\sqrt{\xi_{n}}\right)^{(m-r)} \omega_{r}\left(f_{\alpha}, \xi_{n}\right)_{p} .
\end{aligned}
$$

As $n \rightarrow+\infty$ and $\xi_{n} \rightarrow 0$, by (78) we obtain that $\left\|\widetilde{E}_{r, n}^{[m]}\right\|_{p} \rightarrow 0$ with rates.

One also finds by (78) that

$$
\left\|W_{r, n}^{[m]}(f ; x)-f(x)\right\|_{p, x} \leq \sum_{\tilde{j}=1}^{m}\left|\delta_{\widetilde{j}, r}^{[m]}\right|\left(\sum_{|\alpha|=\widetilde{j}} \frac{\left|\widetilde{c}_{\alpha, n, \tilde{j}}\right|}{\prod_{i=1}^{N} \alpha_{i} !}\left\|f_{\alpha}\right\|_{p}\right)+\text { R.H.S.(78), }
$$

given that $\left\|f_{\alpha}\right\|_{p}<\infty,|\alpha|=\widetilde{j}, \widetilde{j}=1, \ldots, m$.

Assuming that $\xi_{n} \rightarrow 0$, as $n \rightarrow \infty$, we get $\left\|W_{r, n}^{[m]}(f)-f\right\|_{p} \rightarrow 0$, that is $W_{r, n}^{[m]} \rightarrow$ I the unit operator, in $L_{p}$ norm, with rates.

Proof. By Theorem 2.5 we get that $\left|\widetilde{c}_{\alpha, n, \tilde{j}}\right| \leq\left(\frac{\mu}{\sqrt{\pi}}\right)^{N}\left(\sqrt{\xi_{n}}\right)^{m} \leq\left(\frac{\mu}{\sqrt{\pi}}\right)^{N}$ and $\widetilde{c}_{\alpha, n, \tilde{j}} \rightarrow 0$. We use also Theorem 2.3 to uniformly bound

$$
\frac{1}{\left(\sqrt{\pi \xi_{n}}\right)^{N}} \int_{\mathbb{R}^{N}}\left(\left(\prod_{i=1}^{N}\left|s_{i}\right|^{\alpha_{i}}\right)\left(1+\frac{\|s\|_{2}}{\xi_{n}}\right)^{r}\right)^{p} e^{-\frac{\left(\sum_{i=1}^{N} s_{i}^{2}\right)}{\xi_{n}}} d s_{1} \ldots d s_{N} \text {. Then we apply directly Theorem }
$$
3.5.

We continue with an application of

Theorem 3.7. ([3], p. 26) Let $f \in\left(C\left(\mathbb{R}^{N}\right) \cap L_{p}\left(\mathbb{R}^{N}\right)\right) ; N \geq 1 ; p, q>1: \frac{1}{p}+\frac{1}{q}=1$. Assume $\mu_{\xi_{n}}$ probability Borel measure on $\mathbb{R}^{N},\left(\xi_{n}\right)_{n \in \mathbb{N}}>0$ and bounded. Also suppose

$$
\int_{\mathbb{R}^{N}}\left(1+\frac{\|s\|_{2}}{\xi_{n}}\right)^{r p} d \mu_{\xi_{n}}(s)<\infty
$$

Then

$$
\left\|\theta_{r, n}^{[0]}(f)-f\right\|_{p} \leq
$$




$$
\left(\int_{\mathbb{R}^{N}}\left(1+\frac{\|s\|_{2}}{\xi_{n}}\right)^{r p} d \mu_{\xi_{n}}(s)\right)^{\frac{1}{p}} \omega_{r}\left(f, \xi_{n}\right)_{p}
$$

As $\xi_{n} \rightarrow 0$, when $n \rightarrow \infty$, we derive $\left\|\theta_{r, n}^{[0]}(f)-f\right\|_{p} \rightarrow 0$, i.e. $\theta_{r, n}^{[0]} \rightarrow I$, the unit operator, in $L_{p}$ norm.

We give

Theorem 3.8. Let $f \in\left(C\left(\mathbb{R}^{N}\right) \cap L_{p}\left(\mathbb{R}^{N}\right)\right) ; N \geq 1 ; p, q>1: \frac{1}{p}+\frac{1}{q}=1, \xi_{n} \in(0,1], n \in \mathbb{N}$. Then

1)

$$
\begin{gathered}
\left\|W_{r, n}^{[0]}(f)-f\right\|_{p} \leq \\
\left(\frac{2}{\sqrt{\pi}}\right)^{\frac{N}{p}}\left[(1+N)^{r p}+2^{r p} \Gamma^{N}(r p+1,1)\right]^{\frac{1}{p}} \frac{\omega_{r}\left(f, \xi_{n}\right)_{p}}{\left(\sqrt{\xi_{n}}\right)^{r}} .
\end{gathered}
$$

2) Let $r \in \mathbb{N}-\{1\}, 0<\alpha \leq 1$, and consider $f \in\left(C\left(\mathbb{R}^{N}\right) \cap L_{p}\left(\mathbb{R}^{N}\right)\right): \omega_{r}(f, t)_{p} \leq K t^{r-1+\alpha}, \forall$ $t>0$, where $K>0$ is independent of $t$. In this case

$$
\frac{\omega_{r}\left(f, \xi_{n}\right)_{p}}{\left(\sqrt{\xi_{n}}\right)^{r}} \leq K \xi_{n}^{\left(\frac{r}{2}+\alpha-1\right)}
$$

and as $n \rightarrow \infty$ and $\xi_{n} \rightarrow 0$, we obtain

$$
\left\|W_{r, n}^{[0]} f-f\right\|_{p} \rightarrow 0,
$$

i.e. $W_{r, n}^{[0]} \rightarrow I$, the unit operator in $L_{p}$ norm.

Proof. By Theorems 2.4 and 3.7. For $r \geq 2$ we get $\frac{r}{2}+\alpha-1>0$.

We mention

Theorem 3.9. ([3], p. 27) Let $f \in\left(C\left(\mathbb{R}^{N}\right) \cap L_{1}\left(\mathbb{R}^{N}\right)\right) ; N \geq 1$. Assume $\mu_{\xi_{n}}$ probability Borel measure on $\mathbb{R}^{N},\left(\xi_{n}\right)_{n \in \mathbb{N}}>0$ and bounded. Also suppose

$$
\int_{\mathbb{R}^{N}}\left(1+\frac{\|s\|_{2}}{\xi_{n}}\right)^{r} d \mu_{\xi_{n}}(s)<\infty .
$$

Then

$$
\begin{gathered}
\left\|\theta_{r, n}^{[0]}(f)-f\right\|_{1} \leq \\
\left(\int_{\mathbb{R}^{N}}\left(1+\frac{\|s\|_{2}}{\xi_{n}}\right)^{r} d \mu_{\xi_{n}}(s)\right) \omega_{r}\left(f, \xi_{n}\right)_{1} .
\end{gathered}
$$

As $\xi_{n} \rightarrow 0$, we get $\theta_{r, n}^{[0]} \rightarrow I$, in $L_{1}$ norm.

We give 
Theorem 3.10. Let $f \in\left(C\left(\mathbb{R}^{N}\right) \cap L_{1}\left(\mathbb{R}^{N}\right)\right) ; N \geq 1, \xi_{n} \in(0,1], n \in \mathbb{N}$. Then

1)

$$
\left\|W_{r, n}^{[0]}(f)-f\right\|_{1} \leq\left(\frac{2}{\sqrt{\pi}}\right)^{N}\left[(1+N)^{r}+2^{r}\left(\frac{\lfloor e r !\rfloor}{e}\right)^{N}\right] \frac{\omega_{r}\left(f, \xi_{n}\right)_{1}}{\left(\sqrt{\xi_{n}}\right)^{r}} .
$$

2) Let $r \in \mathbb{N}-\{1\}, 0<\alpha \leq 1$, and consider $f \in\left(C\left(\mathbb{R}^{N}\right) \cap L_{1}\left(\mathbb{R}^{N}\right)\right): \omega_{r}(f, t)_{1} \leq K t^{r-1+\alpha}, \forall$ $t>0$, where $K>0$ is independent of $t$. In this case

$$
\frac{\omega_{r}\left(f, \xi_{n}\right)_{1}}{\left(\sqrt{\xi_{n}}\right)^{r}} \leq K \xi_{n}^{\left(\frac{r}{2}+\alpha-1\right)}
$$

and as $n \rightarrow \infty$ and $\xi_{n} \rightarrow 0$, we derive $\left\|W_{r, n}^{[0]}(f)-f\right\|_{1} \rightarrow 0$, i.e. $W_{r, n}^{[0]} \rightarrow I$ in $L_{1}$ norm.

Proof. By Theorems 2.2 and 3.9. Notice $\frac{r}{2}+\alpha-1>0$.

We need

Theorem 3.11. ([3], p. 29) Let $f \in C^{m}\left(\mathbb{R}^{N}\right), m, N \in \mathbb{N}$, with $f_{\alpha} \in L_{1}\left(\mathbb{R}^{N}\right),|\alpha|=m, x \in \mathbb{R}^{N}$. Here, $\mu_{\xi_{n}}$ is a Borel probability measure on $\mathbb{R}^{N}$ for $\xi_{n}>0,\left(\xi_{n}\right)_{n \in \mathbb{N}}$ is a bounded sequence. Suppose for all $\alpha:=\left(\alpha_{1}, \ldots, \alpha_{N}\right), \alpha_{i} \in \mathbb{Z}^{+}, i=1, \ldots, N,|\alpha|:=\sum_{i=1}^{N} \alpha_{i}=m$ that we have

$$
\int_{\mathbb{R}^{N}}\left(\left(\prod_{i=1}^{N}\left|s_{i}\right|^{\alpha_{i}}\right)\left(1+\frac{\|s\|_{2}}{\xi_{n}}\right)^{r}\right) d \mu_{\xi_{n}}(s)<\infty .
$$

For $\widetilde{j}=1, \ldots, m$, and $\alpha:=\left(\alpha_{1}, \ldots, \alpha_{N}\right), \alpha_{i} \in \mathbb{Z}^{+}, i=1, \ldots, N,|\alpha|:=\sum_{i=1}^{N} \alpha_{i}=\widetilde{j}$, call

$$
c_{\alpha, n, \tilde{j}}:=\int_{\mathbb{R}^{N}} \prod_{i=1}^{N} s_{i}^{\alpha_{i}} d \mu_{\xi_{n}}(s) .
$$

Then

$$
\begin{gathered}
\left\|E_{r, n}^{[m]}\right\|_{1}=\left\|\theta_{r, n}^{[m]}(f ; x)-f(x)-\sum_{\widetilde{j}=1}^{m} \delta_{\widetilde{j}, r}^{[m]}\left(\sum_{|\alpha|=\tilde{j}} \frac{c_{\alpha, n, \tilde{j}} f_{\alpha}(x)}{\prod_{i=1}^{N} \alpha_{i} !}\right)\right\|_{1, s} \\
\leq \sum_{|\alpha|=m}\left(\frac{1}{\prod_{i=1}^{N} \alpha_{i} !}\right) \omega_{r}\left(f_{\alpha}, \xi_{n}\right)_{1} \int_{\mathbb{R}^{N}}\left(\prod_{i=1}^{N}\left|s_{i}\right|^{\alpha_{i}}\right)\left(1+\frac{\|s\|_{2}}{\xi_{n}}\right)^{r} d \mu_{\xi_{n}}(s) .
\end{gathered}
$$

As $\xi_{n} \rightarrow 0$, we get $\left\|E_{r, n}^{[m]}\right\|_{1} \rightarrow 0$ with rates. 
From (91) we get

$$
\left\|\theta_{r, n}^{[m]} f-f\right\|_{1} \leq \sum_{\tilde{j}=1}^{m}\left|\delta_{\widetilde{j}, r}^{[m]}\right|\left(\sum_{|\alpha|=\tilde{j}} \frac{\left|c_{\alpha, n, \tilde{j}}\right|}{\prod_{i=1}^{N} \alpha_{i} !}\left\|f_{\alpha}\right\|_{1}\right)+\text { R.H.S.(91), }
$$

given that $\left\|f_{\alpha}\right\|_{1}<\infty,|\alpha|=\widetilde{j}, \widetilde{j}=1, \ldots, m$.

As $n \rightarrow \infty$, assuming $\xi_{n} \rightarrow 0$ and $c_{\alpha, n, \tilde{j}} \rightarrow 0$, we obtain $\left\|\theta_{r, n}^{[m]}(f)-f\right\|_{1} \rightarrow 0$, that is $\theta_{r, n}^{[m]} \rightarrow I$ in $L_{1}$ norm, with rates.

We present

Theorem 3.12. Let $f \in C^{m}\left(\mathbb{R}^{N}\right), m, N \in \mathbb{N}, m>r$, with $f_{\alpha} \in L_{1}\left(\mathbb{R}^{N}\right),|\alpha|=m, x \in \mathbb{R}^{N}$, $\xi_{n} \in(0,1], n \in \mathbb{N}$. For $\widetilde{j}=1, \ldots, m$, and $\alpha:=\left(\alpha_{1}, \ldots, \alpha_{N}\right), \alpha_{i} \in \mathbb{Z}^{+}, i=1, \ldots, N,|\alpha|:=\sum_{i=1}^{N} \alpha_{i}=\widetilde{j}$, call

$$
\widetilde{c}_{\alpha, n, \tilde{j}}:=\frac{1}{\left(\sqrt{\pi \xi_{n}}\right)^{N}} \int_{\mathbb{R}^{N}} \prod_{i=1}^{N} s_{i}^{\alpha_{i}} e^{-\frac{\left(\sum_{i=1}^{N} s_{i}^{2}\right)}{\tilde{\xi}_{n}}} d s_{1} \ldots d s
$$

Then

$$
\begin{gathered}
\left\|\widetilde{E}_{r, n}^{[m]}\right\|_{1}=\left\|W_{r, n}^{[m]}(f ; x)-f(x)-\sum_{\tilde{j}=1}^{m} \delta_{\widetilde{j}, r}^{[m]}\left(\sum_{|\alpha|=\tilde{j}} \frac{\widetilde{c}_{\alpha, n, \tilde{j}} f_{\alpha}(x)}{\prod_{i=1}^{N} \alpha_{i} !}\right)\right\|_{1, x} \leq \\
\left(\frac{2}{\sqrt{\pi}}\right)^{N}\left[(1+N)^{r}+2^{r}\left(\frac{\lfloor e(m+r) !\rfloor}{e}\right)^{N}\right] \sum_{|\alpha|=m}\left(\frac{1}{\prod_{i=1}^{N} \alpha_{i} !}\right)\left(\sqrt{\xi_{n}}\right)^{m-r} \omega_{r}\left(f_{\alpha}, \xi_{n}\right)_{1} \cdot
\end{gathered}
$$

As $\xi_{n} \rightarrow 0$, we get $\left\|\widetilde{E}_{r, n}^{[m]}\right\|_{1} \rightarrow 0$ with rates.

From (94) we get

$$
\left\|W_{r, n}^{[m]} f-f\right\|_{1} \leq \sum_{\widetilde{j}=1}^{m}\left|\delta_{\tilde{j}, r}^{[m]}\right|\left(\sum_{|\alpha|=\widetilde{j}} \frac{\left|\widetilde{c}_{\alpha, n, \tilde{j}}\right|}{\prod_{i=1}^{N} \alpha_{i} !}\left\|f_{\alpha}\right\|_{1}\right)+\text { R.H.S.(94), }
$$

given that $\left\|f_{\alpha}\right\|_{1}<\infty,|\alpha|=\widetilde{j}, \widetilde{j}=1, \ldots, m$.

As $n \rightarrow \infty$, assuming $\xi_{n} \rightarrow 0$, we obtain $\left\|W_{r, n}^{[m]}(f)-f\right\|_{1} \rightarrow 0$, that is $W_{r, n}^{[m]} \rightarrow I$ in $L_{1}$ norm, with rates.

Proof. We apply Theorems 2.1, 2.5 and 3.11. 
3.3. Global Smoothness Preservation and Simultaneous Approximation of $W_{r, n}^{[m]}$. We need

Definition 3.2. ([3], $p$. 34) Let $f \in C\left(\mathbb{R}^{N}\right), N \geq 1, m \in \mathbb{N}$, the mth modulus of smoothness for $1 \leq p \leq \infty$, is given by

$$
\omega_{m}(f ; h)_{p}:=\sup _{\|t\|_{2} \leq h}\left\|\Delta_{t}^{m}(f)\right\|_{p, x}
$$

$h>0$, where

$$
\Delta_{t}^{m} f(x):=\sum_{j=0}^{m}(-1)^{m-j}\left(\begin{array}{c}
m \\
j
\end{array}\right) f(x+j t)
$$

Denote

$$
\omega_{m}(f ; h)_{\infty}=\omega_{m}(f, h) .
$$

Above, $x, t \in \mathbb{R}^{N}$.

We present the related global smoothness preservation result

Theorem 3.13. We assume $W_{r, n}^{[\widetilde{m}]}(f ; x) \in \mathbb{R}, \widetilde{m} \in \mathbb{Z}_{+}, \forall x \in \mathbb{R}$. Let $h>0, f \in C\left(\mathbb{R}^{N}\right), N \geq 1$.

i) Assume $\omega_{m}(f, h)<\infty$. Then

$$
\omega_{m}\left(W_{r, h}^{[\widetilde{m}]} f, h\right) \leq\left(\sum_{\tilde{j}=0}^{r}\left|\alpha_{\tilde{j}, r}^{[\widetilde{m}]}\right|\right) \omega_{m}(f, h) .
$$

ii) Assume $f \in\left(C\left(\mathbb{R}^{N}\right) \cap L_{1}\left(\mathbb{R}^{N}\right)\right)$. Then

$$
\omega_{m}\left(W_{r, h}^{[\widetilde{m}]} f, h\right)_{1} \leq\left(\sum_{\tilde{j}=0}^{r}\left|\alpha_{\tilde{j}, r}^{[\widetilde{m}]}\right|\right) \omega_{m}(f, h)_{1} .
$$

iii) Assume $f \in\left(C\left(\mathbb{R}^{N}\right) \cap L_{p}\left(\mathbb{R}^{N}\right)\right), p>1$. Then

$$
\omega_{m}\left(W_{r, h}^{[\widetilde{m}]} f, h\right)_{p} \leq\left(\sum_{\tilde{j}=0}^{r}\left|\alpha_{\tilde{j}, r}^{[\widetilde{m}]}\right|\right) \omega_{m}(f, h)_{p} .
$$

Proof. Direct application of ([3]) Theorem 3.2, p. 35.

We make

Remark 3.1. Let $r=1, \widetilde{m} \in \mathbb{Z}_{+}$, then $\alpha_{0,1}^{[\widetilde{m}]}=0, \alpha_{1,1}^{[\widetilde{m}]}=1$. Hence

$$
W_{1, n}^{[\widetilde{m}]}(f ; x)=\frac{1}{\left(\sqrt{\pi \xi_{n}}\right)^{N}} \int_{\mathbb{R}^{N}} f(x+s) e^{-\left(\frac{\sum_{i=1}^{N} s_{i}^{2}}{\tilde{\xi} n}\right)} d s_{1} \ldots d s_{N}=: W_{n}(f ; x) .
$$

By Theorem 3.13, we get 
Theorem 3.14. We suppose $W_{n}(f ; x) \in \mathbb{R}, \forall x \in \mathbb{R}$. Let $h>0, f \in C\left(\mathbb{R}^{N}\right), N \geq 1$.

i) Assume $\omega_{m}(f, h)<\infty$. Then

$$
\omega_{m}\left(W_{n} f, h\right) \leq \omega_{m}(f, h) .
$$

ii) Assume $f \in\left(C\left(\mathbb{R}^{N}\right) \cap L_{1}\left(\mathbb{R}^{N}\right)\right)$. Then

$$
\omega_{m}\left(W_{n} f, h\right)_{1} \leq \omega_{m}(f, h)_{1} .
$$

iii) Assume $f \in\left(C\left(\mathbb{R}^{N}\right) \cap L_{p}\left(\mathbb{R}^{N}\right)\right), p>1$. Then

$$
\omega_{m}\left(W_{n} f, h\right)_{p} \leq \omega_{m}(f, h)_{p} .
$$

Next, we get an optimality result

Proposition 3.1. Above inequality (103):

$$
\omega_{m}\left(W_{n} f, h\right) \leq \omega_{m}(f, h)
$$

is sharp, namely it is attained by any

$$
f_{j}^{*}(x)=x_{j}^{m}, j=1, \ldots, N, \quad x=\left(x_{1}, \ldots, x_{j}, \ldots, x_{N}\right) \in \mathbb{R}^{N} .
$$

Proof. Apply Proposition 3.5, p. 38, of [3].

We need

Theorem 3.15. ([3], p. 39) Let $f \in C^{l}\left(\mathbb{R}^{N}\right), l, N \in \mathbb{N}$. Here, $\mu_{\xi_{n}}$ is a Borel probability measure on $\mathbb{R}^{N}, \xi_{n}>0,\left(\xi_{n}\right)_{n \in \mathbb{N}}$ a bounded sequence. Let $\beta:=\left(\beta_{1}, \ldots, \beta_{N}\right), \beta_{i} \in \mathbb{Z}^{+}, i=1, \ldots, N ;|\beta|:=$ $\sum_{i=1}^{N} \beta_{i}=l$. Here $f(x+s j), x, s \in \mathbb{R}^{N}$, is $\mu_{\xi_{n}}$-integrable wrt $s$, for $j=1, \ldots, r$. There exist $\mu_{\xi_{n}}$-integrable functions $h_{i_{1}, j}, h_{\beta_{1}, i_{2}, j}, h_{\beta_{1}, \beta_{2}, i_{3}, j}, \ldots, h_{\beta_{1}, \beta_{2}, \ldots, \beta_{N-1}, i_{N}, j} \geq 0(j=1, \ldots, r)$ on $\mathbb{R}^{N}$ such that

$$
\begin{aligned}
& \left|\frac{\partial^{i_{1}} f(x+s j)}{\partial x_{1}^{i_{1}}}\right| \leq h_{i_{1}, j}(s), \quad i_{1}=1, \ldots, \beta_{1} \\
& \left|\frac{\partial^{\beta_{1}+i_{2}} f(x+s j)}{\partial x_{2}^{i_{2}} \partial x_{1}^{\beta_{1}}}\right| \leq h_{\beta_{1}, i_{2}, j}(s), \quad i_{2}=1, \ldots, \beta_{2}, \\
& \left|\frac{\partial^{\beta_{1}+\beta_{2}+\ldots+\beta_{N-1}+i_{N}} f(x+s j)}{\partial x_{N}^{i_{N}} \partial x_{N-1}^{\beta_{N-1} \ldots \partial x_{2}^{\beta_{2}} \partial x_{1}^{\beta_{1}}}}\right| \leq h_{\beta_{1}, \beta_{2}, \ldots, \beta_{N-1, i_{N}, j}}(s), \quad i_{N}=1, \ldots, \beta_{N},
\end{aligned}
$$

$\forall x, s \in \mathbb{R}^{N}$.

Then, both of the next exist and

$$
\left(\theta_{r, n}^{[\widetilde{m}]}(f ; x)\right)_{\beta}=\theta_{r, n}^{[\widetilde{m}]}\left(f_{\beta} ; x\right), \quad \widetilde{m} \in \mathbb{Z}_{+} .
$$

In particular it holds

$$
\left(W_{r, n}^{[\widetilde{m}]}(f ; x)\right)_{\beta}=W_{r, n}^{[\widetilde{m}]}\left(f_{\beta} ; x\right),
$$


when

$$
d \mu_{\xi_{n}}=\frac{1}{\left(\sqrt{\pi \xi_{n}}\right)^{N}} e^{-\left(\frac{\sum_{i=1}^{N} s_{i}^{2}}{\xi n}\right)} d s_{1} \ldots d s_{N} .
$$

Corollary 3.1. (by Theorem 3.15, $r=1$ ) It holds

$$
\left(W_{n}(f ; x)\right)_{\beta}=W_{n}\left(f_{\beta} ; x\right) \text {. }
$$

We present simultaneous global smoothness results.

Theorem 3.16. Let $h>0$ and the assumptions of Theorem 3.15 are valid for $d \mu_{\xi_{n}}=\frac{1}{\left(\sqrt{\pi \xi_{n}}\right)^{N}} e$ $-\left(\frac{\sum_{i=1}^{N} s_{i}^{2}}{\xi^{2} n}\right)_{d s_{1}}$ Here $\gamma=0, \beta(0=(0, \ldots, 0)), \widetilde{m} \in \mathbb{Z}_{+}$.

i) Assume $\omega_{m}\left(f_{\gamma}, h\right)<\infty$. Then

$$
\omega_{m}\left(\left(W_{r, n}^{[\widetilde{m}]}(f)\right)_{\gamma}, h\right) \leq\left(\sum_{\tilde{j}=0}^{r}\left|\alpha_{\tilde{j}, r}^{[\widetilde{m}]}\right|\right) \omega_{m}\left(f_{\gamma}, h\right) .
$$

ii) Additionally suppose $f_{\gamma} \in L_{1}\left(\mathbb{R}^{N}\right)$. Then

$$
\omega_{m}\left(\left(W_{r, n}^{[\widetilde{m}]}(f)\right)_{\gamma}, h\right)_{1} \leq\left(\sum_{\tilde{j}=0}^{r}\left|\alpha_{\widetilde{j}, r}^{[\widetilde{m}]}\right|\right) \omega_{m}\left(f_{\gamma}, h\right)_{1} .
$$

iii) Additionally suppose $f_{\gamma} \in L_{p}\left(\mathbb{R}^{N}\right), p>1$. Then

$$
\omega_{m}\left(\left(W_{r, n}^{[\widetilde{m}]}(f)\right)_{\gamma}, h\right)_{p} \leq\left(\sum_{\tilde{j}=0}^{r}\left|\alpha_{\widetilde{j}, r}^{[\widetilde{m}]}\right|\right) \omega_{m}\left(f_{\gamma}, h\right)_{p} .
$$

It follows

Corollary 3.2. (to Theorem 3.16) Let $h>0, r=1$ and $\gamma=0, \beta$.

i) Assume $\omega_{m}\left(f_{\gamma}, h\right)<\infty$. Then

$$
\omega_{m}\left(\left(W_{n}(f)\right)_{\gamma}, h\right) \leq \omega_{m}\left(f_{\gamma}, h\right) .
$$

ii) Additionally suppose $f_{\gamma} \in L_{1}\left(\mathbb{R}^{N}\right)$. Then

$$
\omega_{m}\left(\left(W_{n}(f)\right)_{\gamma}, h\right)_{1} \leq \omega_{m}\left(f_{\gamma}, h\right)_{1} .
$$

iii) Additionally suppose $f_{\gamma} \in L_{p}\left(\mathbb{R}^{N}\right), p>1$. Then

$$
\omega_{m}\left(\left(W_{n}(f)\right)_{\gamma}, h\right)_{p} \leq \omega_{m}\left(f_{\gamma}, h\right)_{p} .
$$

Next comes multi-simultaneous approximation. 
Theorem 3.17. Let $f \in C^{m+l}\left(\mathbb{R}^{N}\right), m, l, N \in \mathbb{N}, m>r$. The assumptions of Theorem 3.15 are valid for $d \mu_{\xi_{n}}=\frac{1}{\left(\sqrt{\pi \xi_{n}}\right)^{N}} e^{-\left(\frac{\sum_{i=1}^{N} s_{i}^{2}}{\tilde{\xi} n}\right)} d s_{1} \ldots d s_{N}$. Call $\gamma=0, \beta$. Assume $\left\|f_{\gamma+\alpha}\right\|_{\infty}<\infty$, and let

$$
\widetilde{\mathcal{u}}_{\xi_{n}}(\alpha):=\frac{1}{\left(\sqrt{\pi \xi_{n}}\right)^{N}} \int_{\mathbb{R}^{N}}\left(\prod_{i=1}^{N}\left|s_{i}\right|^{\alpha_{i}}\right)\left(1+\frac{\|s\|_{2}}{\xi_{n}}\right)^{r} e^{-\frac{\left(\sum_{i=1}^{N} s_{i}^{2}\right)}{\xi n}} d s_{1} \ldots d s_{N},
$$

for all $\alpha_{j} \in \mathbb{Z}^{+}, j=1, \ldots, N,|\alpha|:=\sum_{j=1}^{N} \alpha_{j}=m, \xi_{n} \in(0,1]$.

$$
\begin{gathered}
\text { For } \widetilde{j}=1, \ldots, m \text {, and } \alpha:=\left(\alpha_{1}, \ldots, \alpha_{N}\right), \alpha_{i} \in \mathbb{Z}^{+}, i=1, \ldots, N,|\alpha|:=\sum_{i=1}^{N} \alpha_{i}=\widetilde{j}, \text { call } \\
\widetilde{c}_{\alpha, n, \tilde{j}}:=\frac{1}{\left(\sqrt{\pi \xi_{n}}\right)^{N}} \int_{\mathbb{R}^{N}} \prod_{i=1}^{N} s_{i}^{\alpha_{i}} e^{-\frac{\left(\sum_{i=1}^{N} s_{i}^{2}\right)}{\xi_{n}}} d s_{1} \ldots d s_{N} .
\end{gathered}
$$

Then

$$
\begin{gathered}
\left\|\left(W_{r, n}^{[m]}(f ; \cdot)\right)_{\gamma}-f_{\gamma}(\cdot)-\sum_{\widetilde{j}=1}^{m} \delta_{\tilde{j}, r}^{[m]}\left(\sum_{\substack{\alpha_{1}, \ldots, \alpha_{N} \geq 0 ; \\
|\alpha|=\tilde{j}}} \frac{\widetilde{c}_{\alpha, n, \tilde{j}_{\gamma}} f_{\gamma+\alpha}(\cdot)}{\prod_{i=1}^{N} \alpha_{i} !}\right)\right\|_{\infty} \\
\leq \sum_{\substack{\alpha_{1}, \ldots, \alpha_{N} \geq 0 ; \\
|\alpha|=m}} \frac{\left(\omega_{r}\left(f_{\gamma+\alpha}, \xi_{n}\right)\right)}{\left(\prod_{i=1}^{N} \alpha_{i} !\right)} \widetilde{\mathcal{u}}_{\xi_{n}}(\alpha) .
\end{gathered}
$$

Proof. Based on Theorems 3.2, 3.15.

We continue with

Theorem 3.18. Let $f \in C_{B}^{l}\left(\mathbb{R}^{N}\right), l, N \in \mathbb{N}$ (functions l-times continuously differentiable and bounded). The assumptions of Theorem 3.15 are valid for $d \mu_{\xi n}=\frac{1}{\left(\sqrt{\pi \xi_{n}}\right)^{N}} e^{-\left(\frac{\sum_{i=1}^{N} s_{i}^{2}}{\xi_{n}}\right)} d s_{1} \ldots d s_{N}$. Call $\gamma=0, \beta, \xi_{n} \in(0,1]$. Then

1)

$$
\left\|\left(W_{r, n}^{[0]} f\right)_{\gamma}-f_{\gamma}\right\|_{\infty} \leq\left(\frac{2}{\sqrt{\pi}}\right)^{N}\left[(1+N)^{r}+2^{r}\left(\frac{\lfloor e r !\rfloor}{e}\right)^{N}\right] \frac{\omega_{r}\left(f_{\gamma}, \xi_{n}\right)}{\left(\sqrt{\xi_{n}}\right)^{r}} .
$$

2) Let $r \in \mathbb{N}-\{1\}, 0<\alpha \leq 1$, and consider $f_{\gamma}: \omega_{r}\left(f_{\gamma}, t\right) \leq K t^{r-1+\alpha}, \forall t>0$, where $K>0$ is independent of $t$. We get

$$
\frac{\omega_{r}\left(f_{\gamma}, \xi_{n}\right)}{\left(\sqrt{\xi_{n}}\right)^{r}} \leq K \xi_{n}^{\left(\frac{r}{2}+\alpha-1\right)}
$$


and as $n \rightarrow \infty$ and $\xi_{n} \rightarrow 0$, we derive $\left(W_{r, n}^{[0]} f\right)_{\gamma} \rightarrow f_{\gamma}$ uniformly.

Proof. By Theorems 3.4 and 3.15 .

We continue with

Theorem 3.19. Let $f \in C^{m+l}\left(\mathbb{R}^{N}\right), m, l, N \in \mathbb{N}, m>r$. The assumptions of Theorem 3.15 are valid for $d \mu_{\xi_{n}}=\frac{1}{\left(\sqrt{\pi \xi_{n}}\right)^{N}} e^{-\left(\frac{\sum_{i=1}^{N} s_{i}^{2}}{\tilde{\xi} n}\right)} d s_{1} \ldots d s_{N}$. Call $\gamma=0, \beta$. Let $f_{(\gamma+\alpha)} \in L_{p}\left(\mathbb{R}^{N}\right),|\alpha|=m, x \in \mathbb{R}^{N}$, and $p, q>1: \frac{1}{p}+\frac{1}{q}=1, \xi_{n} \in(0,1], n \in \mathbb{N}$. For $\widetilde{j}=1, \ldots, m$, and $\alpha:=\left(\alpha_{1}, \ldots, \alpha_{N}\right), \alpha_{i} \in \mathbb{Z}^{+}$, $i=1, \ldots, N,|\alpha|:=\sum_{i=1}^{N} \alpha_{i}=\widetilde{j}$, call

$$
\widetilde{c}_{\alpha, n, \tilde{j}}:=\frac{1}{\left(\sqrt{\pi \xi_{n}}\right)^{N}} \int_{\mathbb{R}^{N}} \prod_{i=1}^{N} s_{i}^{\alpha_{i}} e^{-\frac{\left(\sum_{i=1}^{N} s_{i}^{2}\right)}{\xi_{n}}} d s_{1} \ldots d s_{N} .
$$

Then

$$
\begin{gathered}
\left\|\left(W_{r, n}^{[m]}(f ; x)\right)_{\gamma}-f_{\gamma}(x)-\sum_{\widetilde{j}=1}^{m} \delta_{\widetilde{j}, r}^{[m]}\left(\sum_{|\alpha|=\widetilde{j}} \frac{\widetilde{c}_{\alpha, n, \tilde{j}} f_{\gamma+\alpha}(x)}{\prod_{i=1}^{N} \alpha_{i} !}\right)\right\|_{p, x} \\
\leq\left(\frac{2}{\sqrt{\pi}}\right)^{\frac{N}{p}}\left(\frac{m}{(q(m-1)+1)^{\frac{1}{q}}}\right)\left[(1+N)^{r p}+2^{r p} \Gamma^{N}((m+r) p+1,1)\right]^{\frac{1}{p}} \\
\\
\left(\sum_{|\alpha|=m} \frac{1}{\prod_{i=1}^{N} \alpha_{i} !}\right)\left(\sqrt{\xi_{n}}\right)^{(m-r)} \omega_{r}\left(f_{\gamma+\alpha}, \xi_{n}\right)_{p} \cdot
\end{gathered}
$$

Proof. By Theorems 3.6, 3.15.

We give also

Theorem 3.20. Let $f \in C^{l}\left(\mathbb{R}^{N}\right), l, N \in \mathbb{N}$. The assumptions of Theorem 3.15 are valid for $d \mu_{\xi_{n}}=$ $\frac{1}{\left(\sqrt{\pi \xi_{n}}\right)^{N}} e^{-\left(\frac{\sum_{i=1}^{N} s_{i}^{2}}{\xi_{n}}\right)} d s_{1} \ldots d s_{N}$. Call $\gamma=0, \beta$. Let $f_{\gamma} \in L_{p}\left(\mathbb{R}^{N}\right), x \in \mathbb{R}^{N} ; p, q>1: \frac{1}{p}+\frac{1}{q}=1$, $\xi_{n} \in(0,1], n \in \mathbb{N}$. Then

1)

$$
\left\|\left(W_{r, n}^{[0]} f\right)_{\gamma}-f_{\gamma}\right\|_{p} \leq
$$




$$
\left(\frac{2}{\sqrt{\pi}}\right)^{\frac{N}{p}}\left[(1+N)^{r p}+2^{r p} \Gamma^{N}(r p+1,1)\right]^{\frac{1}{p}} \frac{\omega_{r}\left(f_{\gamma}, \xi_{n}\right)_{p}}{\left(\sqrt{\xi_{n}}\right)^{r}} .
$$

2) Let $r \in \mathbb{N}-\{1\}, 0<\alpha \leq 1$, and $f_{\gamma}: \omega_{r}\left(f_{\gamma}, t\right)_{p} \leq K t^{r-1+\alpha}, \forall t>0, K>0$ independent of $t$. In this case

$$
\frac{\omega_{r}\left(f_{\gamma}, \xi_{n}\right)_{p}}{\left(\sqrt{\xi_{n}}\right)^{r}} \leq K \xi_{n}^{\left(\frac{r}{2}+\alpha-1\right)}
$$

As $n \rightarrow+\infty$ and $\xi_{n} \rightarrow 0$, then $\left(W_{r, n}^{[0]}(f)\right)_{\gamma} \stackrel{\|\cdot\|_{p}}{\rightarrow} f_{\gamma}$.

Proof. By Theorems 3.8 and 3.15 .

We continue with

Theorem 3.21. Let $f \in C^{l}\left(\mathbb{R}^{N}\right), l, N \in \mathbb{N}$. The assumptions of Theorem 3.15 are valid for $d \mu_{\xi_{n}}=$ $\frac{1}{\left(\sqrt{\pi \xi_{n}}\right)^{N}} e^{-\left(\frac{\sum_{i=1}^{N} s_{i}^{2}}{\xi_{n}}\right)} d s_{1} \ldots d s_{N}$. Call $\gamma=0, \beta$. Let $f_{\gamma} \in L_{1}\left(\mathbb{R}^{N}\right), x \in \mathbb{R}^{N}, \xi_{n} \in(0,1], n \in \mathbb{N}$. Then 1)

$$
\begin{gathered}
\left\|\left(W_{r, n}^{[0]}(f)\right)_{\gamma}-f_{\gamma}\right\|_{1} \leq \\
\left(\frac{2}{\sqrt{\pi}}\right)^{N}\left[(1+N)^{r}+2^{r}\left(\frac{\lfloor e r !\rfloor}{e}\right)^{N}\right] \frac{\omega_{r}\left(f_{\gamma}, \xi_{n}\right)_{1}}{\left(\sqrt{\xi_{n}}\right)^{r}} .
\end{gathered}
$$

2) Let $r \in \mathbb{N}-\{1\}, 0<\alpha \leq 1$, and $f_{\gamma}: \omega_{r}\left(f_{\gamma}, t\right)_{1} \leq K t^{r-1+\alpha}, \forall t>0, K>0$ independent of $t$. In this case

$$
\frac{\omega_{r}\left(f_{\gamma}, \xi_{n}\right)_{1}}{\left(\sqrt{\xi_{n}}\right)^{r}} \leq K \xi_{n}^{\left(\frac{r}{2}+\alpha-1\right)},
$$

and as $n \rightarrow \infty$ and $\xi_{n} \rightarrow 0$, we get $\left(W_{r, n}^{[0]}(f)\right)_{\gamma} \stackrel{\|\cdot\|_{1}}{\rightarrow} f_{\gamma}$.

Proof. By Theorems 3.10 and 3.15.

We continue with

Theorem 3.22. Let $f \in C^{m+l}\left(\mathbb{R}^{N}\right), m, l, N \in \mathbb{N}, m>r$. The assumptions of Theorem 3.15 for $d \mu_{\xi_{n}}=\frac{1}{\left(\sqrt{\pi \xi_{n}}\right)^{N}} e^{-\left(\frac{\sum_{i=1}^{N} s_{i}^{2}}{\xi_{n}}\right)} d s_{1} \ldots d s_{N}$ are valid. Call $\gamma=0, \beta$. Let $f_{(\gamma+\alpha)} \in L_{1}\left(\mathbb{R}^{N}\right),|\alpha|=m$, $x \in \mathbb{R}^{N}, \xi_{n} \in(0,1], n \in \mathbb{N}$. For $\widetilde{j}=1, \ldots, m$, and $\alpha:=\left(\alpha_{1}, \ldots, \alpha_{N}\right), \alpha_{i} \in \mathbb{Z}^{+}, i=1, \ldots, N$, 
$|\alpha|:=\sum_{i=1}^{N} \alpha_{i}=\widetilde{j}$, call

$$
\widetilde{c}_{\alpha, n, \tilde{j}}:=\frac{1}{\left(\sqrt{\pi \xi_{n}}\right)^{N}} \int_{\mathbb{R}^{N}} \prod_{i=1}^{N} s_{i}^{\alpha_{i}} e^{-\frac{\left(\sum_{i=1}^{N} s_{i}^{2}\right)}{\tilde{\xi} n}} d s_{1} \ldots d s_{N} .
$$

Then

$$
\begin{gathered}
\left\|\left(W_{r, n}^{[m]}(f ; x)\right)_{\gamma}-f_{\gamma}(x)-\sum_{\tilde{j}=1}^{m} \delta_{\tilde{j}, r}^{[m]}\left(\sum_{|\alpha|=\tilde{j}} \frac{\widetilde{c}_{\alpha, n, \tilde{j}} f_{(\gamma+\alpha)}(x)}{\prod_{i=1}^{N} \alpha_{i} !}\right)\right\|_{1, x} \\
\leq\left(\frac{2}{\sqrt{\pi}}\right)^{N}\left(\sum_{|\alpha|=m}\left(\frac{1}{\prod_{i=1}^{N} \alpha_{i} !}\right) \omega_{r}\left(f_{\gamma+\alpha}, \xi_{n}\right)_{1}\right] \\
\left(\sqrt{\xi_{n}}\right)^{m-r}\left[(1+N)^{r}+2^{r}\left(\frac{\lfloor e(m+r) !\rfloor}{e}\right)^{N}\right] .
\end{gathered}
$$

Proof. By Theorems 3.12, 3.15.

3.4. Voronovskaya Asymptotic Expansions for $W_{r, n}^{[m]}$. Here we will apply the following general theorem.

Theorem 3.23. Let $f \in C^{m}\left(\mathbb{R}^{N}\right), m, N \in \mathbb{N}$, with all $\left\|f_{\alpha}\right\|_{\infty} \leq M, M>0$, all $\alpha:|\alpha|=m$. Let $\xi_{n}>0,\left(\xi_{n}\right)_{n \in \mathbb{N}}$ bounded sequence, $\mu_{\xi_{n}}$ probability Borel measures on $\mathbb{R}^{N}$.

Call $c_{\alpha, n, \tilde{j}}:=\int_{\mathbb{R}^{N}}\left(\prod_{i=1}^{N} s_{i}^{\alpha_{i}}\right) d \mu_{\xi_{n}}(s)$, all $|\alpha|=\widetilde{j}=1, \ldots, m-1$. Suppose

$$
\left(\sqrt{\xi_{n}}\right)^{-m} \int_{\mathbb{R}^{N}}\left(\prod_{i=1}^{N}\left|s_{i}\right|^{\alpha_{i}}\right) d \mu_{\xi_{n}}(s) \leq \rho,
$$

all $\alpha:|\alpha|=m, \rho>0$, for any such $\left(\xi_{n}\right)_{n \in \mathbb{N}^{N}}$ Also $0<\gamma \leq 1, x \in \mathbb{R}^{N}$. Then

$$
\theta_{r, n}^{[m]}(f ; x)-f(x)=\sum_{\tilde{j}=1}^{m-1} \delta_{\widetilde{j}, r}^{[m]}\left(\sum_{|\alpha|=\tilde{j}} \frac{c_{\alpha, n, \tilde{j}} f_{\alpha}(x)}{\left(\prod_{i=1}^{N} \alpha_{i} !\right)}\right)+0\left(\left(\sqrt{\xi_{n}}\right)^{m-\gamma}\right) .
$$

When $m=1$, the sum collapses.

Above we assume $\theta_{r, n}^{[\mathrm{m}]}(f ; x) \in \mathbb{R}, \forall x \in \mathbb{R}^{N}$.

Proof. The proof of Theorem 3.23 is the same as the proof of Theorem 4.2, p. 53 of [3], just replace there $\xi_{n}$ by $\sqrt{\xi_{n}}$. 
We give

Theorem 3.24. Let $f \in C^{m}\left(\mathbb{R}^{N}\right), m, N \in \mathbb{N}$, with all $\left\|f_{\alpha}\right\|_{\infty} \leq M, M>0$, all $\alpha:|\alpha|=m$, $\xi_{n} \in(0,1]$, and $d \mu_{\xi_{n}}=\frac{1}{\left(\sqrt{\pi \xi_{n}}\right)^{N}} e^{-\left(\frac{\sum_{i=1}^{N} s_{i}^{2}}{\xi^{n} n}\right)} d s_{1} \ldots d s_{N}$ on $\mathbb{R}^{N}$. Assume $W_{r, n}^{[m]}(f ; x) \in \mathbb{R}, \forall x \in \mathbb{R}^{N}$. Call $\widetilde{c}_{\alpha, n, \tilde{j}}=\frac{1}{\left(\sqrt{\pi \xi_{n}}\right)^{N}} \int_{\mathbb{R}^{N}}\left(\prod_{i=1}^{N} s_{i}^{\alpha_{i}}\right) e^{-\left(\frac{\sum_{i=1}^{N} s_{i}^{2}}{\tilde{\xi} n}\right)} d s_{1} \ldots d s_{N}$, all $|\alpha|=\widetilde{j}=1, \ldots, m-1$. Let $0<\gamma \leq 1$, $x \in \mathbb{R}^{N}$. Then

$$
W_{r, n}^{[m]}(f ; x)-f(x)=\sum_{\tilde{j}=1}^{m-1} \delta_{\widetilde{j}, r}^{[m]}\left(\sum_{|\alpha|=\tilde{j}} \frac{\widetilde{c}_{\alpha, n, \widetilde{j}^{\prime}} f_{\alpha}(x)}{\left(\prod_{i=1}^{N} \alpha_{i} !\right)}\right)+0\left(\left(\sqrt{\xi_{n}}\right)^{m-\gamma}\right) .
$$

When $m=1$, the sum collapses.

Proof. By Theorems 2.5 and 3.23. Here $\rho=\left(\frac{\mu}{\sqrt{\pi}}\right)^{N}$.

Corollary 3.3. (to Theorem 3.24) Let $f \in C^{1}\left(\mathbb{R}^{N}\right), N \geq 1$, with all $\left\|\frac{\partial f}{\partial x_{i}}\right\|_{\infty} \leq M, M>0, i=$ $1, \ldots, N$. Let $0<\gamma \leq 1, x \in \mathbb{R}^{N}$. Then

$$
W_{r, n}^{[1]}(f ; x)-f(x)=0\left(\left(\sqrt{\xi_{n}}\right)^{1-\gamma}\right) .
$$

Above we assume $W_{r, n}^{[1]}(f ; x) \in \mathbb{R}, \forall x \in \mathbb{R}^{N}$.

Proof. By Theorems 2.5 and 3.23, here it is $\rho=1$.

Corollary 3.4. (to Theorem 3.24) Let $f \in C^{2}\left(\mathbb{R}^{2}\right)$, with all $\left\|\frac{\partial^{2} f}{\partial x_{1}^{2}}\right\|_{\infty},\left\|\frac{\partial^{2} f}{\partial x_{2}^{2}}\right\|_{\infty},\left\|\frac{\partial^{2} f}{\partial x_{1} \partial x_{2}}\right\|_{\infty} \leq M$, $M>0, \xi_{n} \in(0,1], n \in \mathbb{N}$. Call

$$
c_{1}=\int_{\mathbb{R}^{2}} s_{1} d \mu_{\xi_{n}}(s), \quad c_{2}=\int_{\mathbb{R}^{2}} s_{2} d \mu_{\xi_{n}}(s),
$$

where

$$
d \mu_{\xi n}=\frac{1}{\sqrt{\pi \xi_{n}}} e^{-\frac{\left(s_{1}^{2}+s_{2}^{2}\right)}{\xi_{n}}} d s_{1} d s_{2}
$$

Let $0<\gamma \leq 1, x \in \mathbb{R}^{2}$. Then

$$
W_{r, n}^{[2]}(f ; x)-f(x)=\left(\sum_{j=1}^{r} \alpha_{j, r}^{[2]} j\right)\left(c_{1} \frac{\partial f}{\partial x_{1}}(x)+c_{2} \frac{\partial f}{\partial x_{2}}(x)\right)+0\left(\left(\sqrt{\xi_{n}}\right)^{2-\gamma}\right) .
$$

Proof. By Theorems 2.5 and 3.23, here it is $\rho=1$. 
We also give

Theorem 3.25. Let $f \in C^{m+l}\left(\mathbb{R}^{N}\right), m, l, N \in \mathbb{N}$. Assumptions of Theorem 3.15 are valid for $d \mu_{\xi_{n}}(s)=\frac{1}{\left(\sqrt{\pi \xi_{n}}\right)^{N}} e^{-\left(\frac{\sum_{i=1}^{N} s_{i}^{2}}{\xi_{n}}\right)} d s_{1} \ldots d s_{N}$. Call $\gamma=0, \beta$. Suppose $\left\|f_{\gamma+\alpha}\right\|_{\infty} \leq M, M>0$, for all $\alpha:|\alpha|=m, \xi_{n} \in(0,1], n \in \mathbb{N}$.

Call $\widetilde{c}_{\alpha, n, \tilde{j}}:=\frac{1}{\left(\sqrt{\pi \xi_{n}}\right)^{N}} \int_{\mathbb{R}^{N}}\left(\prod_{i=1}^{N} s_{i}^{\alpha_{i}}\right) e^{-\left(\frac{\sum_{i=1}^{N} s_{i}^{2}}{\xi_{n}}\right)} d s_{1} \ldots d s_{N}$, all $|\alpha|=\widetilde{j}=1, \ldots, m-1 ; 0<\gamma \leq 1$, $x \in \mathbb{R}^{N}$. Then

$$
\left(W_{r, n}^{[m]}(f ; x)\right)_{\gamma}-f_{\gamma}(x)=\sum_{\tilde{j}=1}^{m-1} \delta_{\widetilde{j}, r}^{[m]}\left(\sum_{|\alpha|=\widetilde{j}} \frac{\widetilde{c}_{\alpha, n, \tilde{j}^{\prime}} f_{\gamma+\alpha}(x)}{\left(\prod_{i=1}^{N} \alpha_{i} !\right)}\right)+0\left(\left(\sqrt{\tilde{\xi}_{n}}\right)^{m-\gamma}\right) .
$$

When $m=1$, the sum collapses.

Proof. Use of Theorem 2.5 and Theorem 4.6, p. 54 of [3], which is true also for $\sqrt{\xi_{n}}$ instead of $\xi_{n}$ in the main assumption and conclusion. Here it is $\rho=\left(\frac{\mu}{\sqrt{\pi}}\right)^{N}$.

\subsection{Simultaneous Approximation by multivariate complex $W_{r, n}^{[m]}$. We make}

Remark 3.2. We consider here complex valued Borel measurable functions $f: \mathbb{R}^{N} \rightarrow \mathbb{C}$ such that $f=f_{1}+i f_{2}, i=\sqrt{-1}$, where $f_{1}, f_{2}: \mathbb{R}^{N} \rightarrow \mathbb{R}$ are implied to be real valued Borel measurable functions.

We define the multivariate complex Gauss-Weierstrass singular operators

$$
W_{r, n}^{[m]}(f ; x):=W_{r, n}^{[m]}\left(f_{1} ; x\right)+i W_{r, n}^{[m]}\left(f_{2} ; x\right), \quad x \in \mathbb{R}^{N} .
$$

We assume that $W_{r, n}^{[m]}\left(f_{j} ; x\right) \in \mathbb{R}, \forall x \in \mathbb{R}^{N}, j=1,2$.

One notices easily that

$$
\left|W_{r, n}^{[m]}(f ; x)-f(x)\right| \leq\left|W_{r, n}^{[m]}\left(f_{1} ; x\right)-f_{1}(x)\right|+\left|W_{r, n}^{[m]}\left(f_{2} ; x\right)-f_{2}(x)\right|
$$

also

$$
\left\|W_{r, n}^{[m]}(f ; x)-f(x)\right\|_{\infty, x} \leq\left\|W_{r, n}^{[m]}\left(f_{1} ; x\right)-f_{1}(x)\right\|_{\infty, x}+\left\|W_{r, n}^{[m]}\left(f_{2} ; x\right)-f_{2}(x)\right\|_{\infty, x}
$$

and

$$
\left\|W_{r, n}^{[m]}(f)-f\right\|_{p} \leq\left\|W_{r, n}^{[m]}\left(f_{1}\right)-f_{1}\right\|_{p}+\left\|W_{r, n}^{[m]}\left(f_{2}\right)-f_{2}\right\|_{p^{\prime}} p \geq 1 .
$$

Furthermore, it holds

$$
f_{\alpha}(x)=f_{1, \alpha}(x)+i f_{2, \alpha}(x),
$$

where $\alpha$ denotes a partial derivative of any order and arrangement. 
We give

Theorem 3.26. Let $f: \mathbb{R}^{N} \rightarrow \mathbb{C}, N \geq 1$, such that $f=f_{1}+i f_{2}, j=1,2$. Here $m \in \mathbb{N}, f_{j} \in$ $C^{m}\left(\mathbb{R}^{N}\right), x \in \mathbb{R}^{N}$. Assume $\left\|\frac{\partial^{m} f_{j}(\cdot,, \ldots, \cdot)}{\partial x_{1}^{\alpha_{1}} \ldots \partial x_{N}^{\alpha_{N}}}\right\|_{\infty}<\infty$, for all $\alpha_{i} \in \mathbb{Z}^{+}, i=1, \ldots, N:|\alpha|:=\sum_{i=1}^{N} \alpha_{i}=m$, $m>r$. Here $\xi_{n} \in(0,1], n \in \mathbb{N}$. For all $\alpha:=\left(\alpha_{1}, \ldots, \alpha_{N}\right), \alpha_{i} \in \mathbb{Z}^{+}, i=1, \ldots, N,|\alpha|:=\sum_{i=1}^{N} \alpha_{i}=m$ we denote

$$
\widetilde{u}_{\xi_{n}}(\alpha):=\frac{1}{\left(\sqrt{\pi \xi_{n}}\right)^{N}} \int_{\mathbb{R}^{N}}\left(\prod_{i=1}^{N}\left|s_{i}\right|^{\alpha_{i}}\right)\left(1+\frac{\|s\|_{2}}{\xi_{n}}\right)^{r} e^{-\left(\frac{\sum_{i=1}^{N} s_{i}^{2}}{\xi_{n}}\right)} d s_{1} \ldots d s_{N} .
$$

For $\widetilde{j}=1, \ldots, m$, and $\alpha:=\left(\alpha_{1}, \ldots, \alpha_{N}\right), \alpha_{i} \in \mathbb{Z}^{+}, i=1, \ldots, N,|\alpha|:=\sum_{i=1}^{N} \alpha_{i}=\widetilde{j}$, call

$$
\widetilde{c}_{\alpha, n}:=\widetilde{c}_{\alpha, n, \widetilde{j}}:=\frac{1}{\left(\sqrt{\pi \xi_{n}}\right)^{N}} \int_{\mathbb{R}^{N}} \prod_{i=1}^{N} s_{i}^{\alpha_{i}} e^{-\left(\frac{\sum_{i=1}^{N} s_{i}^{2}}{\xi_{n}}\right)} d s_{1} \ldots d s_{N} .
$$

Then

$$
\begin{aligned}
& \left\|W_{r, n}^{[m]}(f ; x)-f(x)-\sum_{\tilde{j}=1}^{m} \delta_{\widetilde{j}, r}^{[m]}\left(\sum_{\substack{\alpha_{1}, \ldots, \alpha_{N} \geq 0 ; \\
|\alpha|=\tilde{j}}} \frac{\widetilde{c}_{\alpha, n, \tilde{j}} f_{\alpha}(x)}{\prod_{i=1}^{N} \alpha_{i} !}\right)\right\|_{\infty, x} \\
& \leq \sum_{\substack{\alpha_{1}, \ldots, \alpha_{N} \geq 0 ; \\
|\alpha|=m}} \frac{\left(\omega_{r}\left(f_{1, \alpha}, \xi_{n}\right)+\omega_{r}\left(f_{2, \alpha}, \xi_{n}\right)\right)}{\left(\prod_{i=1}^{N} \alpha_{i} !\right)} \widetilde{u}_{\xi_{n}}(\alpha) \text {. }
\end{aligned}
$$

Proof. By Theorem 3.2.

We proceed with

Theorem 3.27. Let $f: \mathbb{R}^{N} \rightarrow \mathbb{C}: f=f_{1}+i f_{2}, N \geq 1, j=1,2$. Here $f_{j} \in C_{B}\left(\mathbb{R}^{N}\right)$ uniformly continuous, $\xi_{n} \in(0,1]$. Then

1)

$$
\left\|W_{r, n}^{[0]} f-f\right\|_{\infty} \leq\left(\frac{2}{\sqrt{\pi}}\right)^{N}\left[(1+N)^{r}+2^{r}\left(\frac{\lfloor\text { er } !\rfloor}{e}\right)^{N}\right] \frac{\left(\omega_{r}\left(f_{1}, \xi_{n}\right)+\omega_{r}\left(f_{2}, \xi_{n}\right)\right)}{\left(\sqrt{\xi_{n}}\right)^{r}},
$$

where $r, n \in \mathbb{N}$.

2) Let $r \in \mathbb{N}-\{1\}, 0<\alpha \leq 1$, and $\omega_{r}\left(f_{j}, t\right) \leq K t^{r-1+\alpha}, \forall t>0, K>0$ is independent of $t$, $j=1,2$. We get

$$
\frac{\omega_{r}\left(f_{j}, \xi_{n}\right)}{\left(\sqrt{\xi_{n}}\right)^{r}} \leq K \xi_{n}^{\left(\frac{r}{2}+\alpha-1\right)}, j=1,2
$$


and as $n \rightarrow \infty$ and $\xi_{n} \rightarrow 0$, we get $\left\|W_{r, n}^{[0]}(f)-f\right\|_{\infty} \rightarrow 0$.

Proof. Use of Theorem 3.4.

Next comes multi-simultaneous approximation

Theorem 3.28. Let $f: \mathbb{R}^{N} \rightarrow \mathbb{C}: f=f_{1}+i f_{2}, j=1,2$. Here $f_{j} \in C^{m+l}\left(\mathbb{R}^{N}\right), m, l, N \in \mathbb{N}, m>r$. The assumptions of Theorem 3.15 are valid for

$$
\begin{gathered}
d \mu_{\xi_{n}}=\frac{1}{\left(\sqrt{\pi \xi_{n}}\right)^{N}} e^{-\left(\frac{\sum_{i=1}^{N} s_{i}^{2}}{\xi_{n}}\right)} d s_{1} \ldots d s_{N} \text { and } f_{j} . \text { Call } \gamma=0, \beta . \text { Assume }\left\|f_{j, \gamma+\alpha}\right\|_{\infty}<\infty \text {, and let } \\
\widetilde{u}_{\xi_{n}}(\alpha):=\frac{1}{\left(\sqrt{\pi \xi_{n}}\right)^{N}} \int_{\mathbb{R}^{N}}\left(\prod_{i=1}^{N}\left|s_{i}\right|^{\alpha_{i}}\right)\left(1+\frac{\|s\|_{2}}{\xi_{n}}\right)^{r} e^{-\left(\frac{\sum_{i=1}^{N} s_{i}^{2}}{\xi_{n}}\right)} d s_{1} \ldots d s_{N} .
\end{gathered}
$$

for all $\alpha_{i} \in \mathbb{Z}^{+}, i=1, \ldots, N:|\alpha|:=\sum_{i=1}^{N} \alpha_{i}=m, \xi_{n} \in(0,1]$.

$$
\begin{gathered}
\text { For } \widetilde{j}=1, \ldots, m \text {, and } \alpha:=\left(\alpha_{1}, \ldots, \alpha_{N}\right), \alpha_{i} \in \mathbb{Z}^{+}, i=1, \ldots, N,|\alpha|:=\sum_{i=1}^{N} \alpha_{i}=\widetilde{j}, \text { call } \\
\widetilde{c}_{\alpha, n, \tilde{j}}:=\frac{1}{\left(\sqrt{\pi \xi_{n}}\right)^{N}} \int_{\mathbb{R}^{N}} \prod_{i=1}^{N} s_{i}^{\alpha_{i}} e^{-\left(\frac{\sum_{i=1}^{N} s_{i}^{2}}{\tilde{\xi} n}\right)} d s_{1} \ldots d s_{N} .
\end{gathered}
$$

Then

$$
\begin{gathered}
\left\|\left(W_{r, n}^{[m]}(f ; \cdot)\right)_{\gamma}-f_{\gamma}(\cdot)-\sum_{\widetilde{j}=1}^{m} \delta_{\widetilde{j}, r}^{[m]}\left(\sum_{\substack{\alpha_{1}, \ldots, \alpha_{N} \geq 0 ; \\
|\alpha|=\widetilde{j}}} \frac{\widetilde{c}_{\alpha, n, \tilde{j}} f_{\gamma+\alpha}(\cdot)}{\prod_{i=1}^{N} \alpha_{i} !}\right)\right\|_{\infty} \\
\leq \sum_{\substack{\alpha_{1}, \ldots, \alpha_{N} \geq 0 ; \\
|\alpha|=m}} \frac{\left(\omega_{r}\left(f_{1, \gamma+\alpha}, \xi_{n}\right)+\omega_{r}\left(f_{2, \gamma+\alpha}, \xi_{n}\right)\right)}{\left(\prod_{i=1}^{N} \alpha_{i} !\right)} \widetilde{u}_{\xi_{n}}(\alpha) .
\end{gathered}
$$

Proof. Based on Theorems 3.15, 3.17.

We continue with

Theorem 3.29. Let $f: \mathbb{R}^{N} \rightarrow \mathbb{C}: f=f_{1}+i f_{2}, j=1,2$. Here $f_{j} \in C_{B}^{l}\left(\mathbb{R}^{N}\right), l, N \in \mathbb{N}$ (functions l-times continuously differentiable and bounded). The assumptions of Theorem 3.15 are valid for $d \mu_{\xi_{n}}=\frac{1}{\left(\sqrt{\pi \xi_{n}}\right)^{N}} e^{-\left(\frac{\sum_{i=1}^{N} s_{i}^{2}}{\xi_{n}}\right)} d s_{1} \ldots d s_{N}$ and $f_{j}, j=1,2$. Call $\gamma=0, \beta, \xi_{n} \in(0,1]$. Then 
1)

$$
\begin{gathered}
\left\|\left(W_{r, n}^{[0]} f\right)_{\gamma}-f_{\gamma}\right\|_{\infty} \leq \\
\left(\frac{2}{\sqrt{\pi}}\right)^{N}\left[(1+N)^{r}+2^{r}\left(\frac{\lfloor e r !\rfloor}{e}\right)^{N}\right] \frac{\left(\omega_{r}\left(f_{1, \gamma}, \xi_{n}\right)+\omega_{r}\left(f_{2, \gamma}, \xi_{n}\right)\right)}{\left(\sqrt{\xi_{n}}\right)^{r}} .
\end{gathered}
$$

2) Let $r \in \mathbb{N}-\{1\}, 0<\alpha \leq 1$, and consider $f_{j, \gamma}: \omega_{r}\left(f_{j, \gamma}, t\right) \leq K t^{r-1+\alpha}, \forall t>0, j=1,2$, where $K>0$ is independent of $t$. We get

$$
\frac{\omega_{r}\left(f_{j, \gamma}, \xi_{n}\right)}{\left(\sqrt{\xi_{n}}\right)^{r}} \leq K \xi_{n}^{\left(\frac{r}{2}+\alpha-1\right)}
$$

$j=1,2$, and as $n \rightarrow \infty$ and $\xi_{n} \rightarrow 0$, we find $\left(W_{r, n}^{[0]} f\right)_{\gamma} \rightarrow f_{\gamma}$ uniformly.

Proof. By Theorems 3.15 and 3.18 .

We proceed with $L_{p}$ approximations

Theorem 3.30. Let $f: \mathbb{R}^{N} \rightarrow \mathbb{C}: f=f_{1}+i f_{2}, j=1,2$. Here $f_{j} \in C^{m}\left(\mathbb{R}^{N}\right), m \in \mathbb{N}, m>r$, $N \geq 1$, with $f_{j, \alpha} \in L_{p}\left(\mathbb{R}^{N}\right),|\alpha|=m, x \in \mathbb{R}^{N}$. Let $p, q>1: \frac{1}{p}+\frac{1}{q}=1, \xi_{n} \in(0,1]$. For $\widetilde{j}=1, \ldots, m$, and $\alpha:=\left(\alpha_{1}, \ldots, \alpha_{N}\right), \alpha_{i} \in \mathbb{Z}^{+}, i=1, \ldots, N,|\alpha|:=\sum_{i=1}^{N} \alpha_{i}=\widetilde{j}$, call

$$
\widetilde{c}_{\alpha, n, \tilde{j}}=\frac{1}{\left(\sqrt{\pi \xi_{n}}\right)^{N}} \int_{\mathbb{R}^{N}} \prod_{i=1}^{N} s_{i}^{\alpha_{i}} e^{-\frac{\left(\sum_{i=1}^{N} s_{i}^{2}\right)}{\xi^{2} n}} d s_{1} \ldots d s .
$$

Then

$$
\begin{gathered}
\left\|W_{r, n}^{[m]}(f ; x)-f(x)-\sum_{\tilde{j}=1}^{m} \delta_{\widetilde{j}, r}^{[m]}\left(\sum_{|\alpha|=\tilde{j}} \frac{\widetilde{c}_{\alpha, n, \tilde{j}} f_{\alpha}(x)}{\left(\prod_{i=1}^{N} \alpha_{i} !\right)}\right)\right\|_{p, x} \\
\leq\left(\frac{2}{\sqrt{\pi}}\right)^{\frac{N}{p}}\left(\frac{m}{(q(m-1)+1)^{\frac{1}{q}}}\right)\left[(1+N)^{r p}+2^{r p} \Gamma^{N}((m+r) p+1,1)\right]^{\frac{1}{p}} \\
\\
\left(\sum_{|\alpha|=m} \frac{1}{\prod_{i=1}^{N} \alpha_{i} !}\right)\left(\sqrt{\xi_{n}}\right)^{(m-r)}\left[\omega_{r}\left(f_{1, \alpha}, \xi_{n}\right)_{p}+\omega_{r}\left(f_{2, \alpha}, \xi_{n}\right)_{p}\right] .
\end{gathered}
$$

Proof. By Theorem 3.6.

We continue with 
Theorem 3.31. Let $f: \mathbb{R}^{N} \rightarrow \mathbb{C}: f=f_{1}+i f_{2}, j=1$,2. Here $f_{j} \in\left(C\left(\mathbb{R}^{N}\right) \cap L_{p}\left(\mathbb{R}^{N}\right)\right) ; N \geq 1$; $p, q>1: \frac{1}{p}+\frac{1}{q}=1, \xi_{n} \in(0,1], n \in \mathbb{N}$. Then

1)

$$
\begin{gathered}
\left\|W_{r, n}^{[0]}(f)-f\right\|_{p} \leq \\
\left(\frac{2}{\sqrt{\pi}}\right)^{\frac{N}{p}}\left[(1+N)^{r p}+2^{r p} \Gamma^{N}(r p+1,1)\right]^{\frac{1}{p}} \frac{\left(\omega_{r}\left(f_{1}, \xi_{n}\right)_{p}+\omega_{r}\left(f_{2}, \xi_{n}\right)_{p}\right)}{\left(\sqrt{\xi_{n}}\right)^{r}} .
\end{gathered}
$$

2) Let $r \in \mathbb{N}-\{1\}, 0<\alpha \leq 1$, and assume that $\omega_{r}\left(f_{j}, t\right)_{p} \leq K t^{r-1+\alpha}, \forall t>0, j=1,2$, where $K>0$ is independent of $t$. In this case

$$
\frac{\omega_{r}\left(f_{j}, \xi_{n}\right)_{p}}{\left(\sqrt{\xi_{n}}\right)^{r}} \leq K \xi_{n}^{\left(\frac{r}{2}+\alpha-1\right)}, j=1,2,
$$

and as $n \rightarrow \infty$ and $\xi_{n} \rightarrow 0$, we get $\left\|W_{r, n}^{[0]} f-f\right\|_{p} \rightarrow 0$.

Proof. By Theorem 3.8 .

We also give

Theorem 3.32. Let $f: \mathbb{R}^{N} \rightarrow \mathbb{C}: f=f_{1}+i f_{2}, j=1$,2. Here $f_{j} \in\left(C\left(\mathbb{R}^{N}\right) \cap L_{1}\left(\mathbb{R}^{N}\right)\right) ; N \geq 1$, $\xi_{n} \in(0,1], n \in \mathbb{N}$. Then

1)

$$
\begin{gathered}
\left\|W_{r, n}^{[0]}(f)-f\right\|_{1} \leq \\
\left(\frac{2}{\sqrt{\pi}}\right)^{N}\left[(1+N)^{r}+2^{r}\left(\frac{\lfloor e r !\rfloor}{e}\right)^{N}\right] \frac{\left[\omega_{r}\left(f_{1}, \xi_{n}\right)_{1}+\omega_{r}\left(f_{2}, \xi_{n}\right)_{1}\right]}{\left(\sqrt{\xi_{n}}\right)^{r}} .
\end{gathered}
$$

2) Let $r \in \mathbb{N}-\{1\}, 0<\alpha \leq 1$, and assume for $j=1$, 2 that $\omega_{r}\left(f_{j}, t\right)_{1} \leq K t^{r-1+\alpha}, \forall t>0, K>0$ independent of $t$. We find that

$$
\frac{\omega_{r}\left(f_{j}, \xi_{n}\right)_{1}}{\left(\sqrt{\xi_{n}}\right)^{r}} \leq K \xi_{n}^{\left(\frac{r}{2}+\alpha-1\right)}, j=1,2,
$$

and as $n \rightarrow \infty$ and $\xi_{n} \rightarrow 0$, we derive $\left\|W_{r, n}^{[0]}(f)-f\right\|_{1} \rightarrow 0$.

Proof. By Theorem 3.10.

We further present

Theorem 3.33. Let $f: \mathbb{R}^{N} \rightarrow \mathbb{C}: f=f_{1}+i f_{2}, j=1,2$. Here $f_{j} \in C^{m}\left(\mathbb{R}^{N}\right), m, N \in \mathbb{N}$, with $f_{j, \alpha} \in L_{1}\left(\mathbb{R}^{N}\right),|\alpha|=m, x \in \mathbb{R}^{N}, \xi_{n} \in(0,1], n \in \mathbb{N}, m>r$. For $\widetilde{j}=1, \ldots, m$, and $\alpha:=\left(\alpha_{1}, \ldots, \alpha_{N}\right)$, 


$$
\begin{aligned}
\alpha_{i} \in \mathbb{Z}^{+}, i=1, \ldots, N,|\alpha| & :=\sum_{i=1}^{N} \alpha_{i}=\widetilde{j}, \text { call } \\
& \widetilde{c}_{\alpha, n, \tilde{j}}:=\frac{1}{\left(\sqrt{\pi \xi_{n}}\right)^{N}} \int_{\mathbb{R}^{N}} \prod_{i=1}^{N} s_{i}^{\alpha_{i}} e^{-\frac{\left(\sum_{i=1}^{N} s_{i}^{2}\right)}{\xi_{n}}} d s_{1} \ldots d s .
\end{aligned}
$$

Then

$$
\begin{gathered}
\left\|W_{r, n}^{[m]}(f ; x)-f(x)-\sum_{\tilde{j}=1}^{m} \delta_{\widetilde{j}, r}^{[m]}\left(\sum_{|\alpha|=\tilde{j}} \frac{\widetilde{c}_{\alpha, n, \tilde{j}} f_{\alpha}(x)}{\prod_{i=1}^{N} \alpha_{i} !}\right)\right\|_{1, x} \\
\leq\left(\frac{2}{\sqrt{\pi}}\right)^{N}\left[(1+N)^{r}+2^{r}\left(\frac{\lfloor e(m+r) !\rfloor}{e}\right)^{N}\right] \sum_{|\alpha|=m}\left(\frac{1}{\prod_{i=1}^{N} \alpha_{i} !}\right) \\
\left(\sqrt{\xi_{n}}\right)^{m-r}\left[\omega_{r}\left(f_{1, \alpha}, \xi_{n}\right)_{1}+\omega_{r}\left(f_{2, \alpha}, \xi_{n}\right)_{1}\right] .
\end{gathered}
$$

Proof. By Theorem 3.12.

We continue with simultaneous $L_{p}$ approximations.

Theorem 3.34. Let $f: \mathbb{R}^{N} \rightarrow \mathbb{C}: f=f_{1}+i f_{2}, j=1,2$. Here $f_{j} \in C^{m+l}\left(\mathbb{R}^{N}\right), m, l, N \in \mathbb{N}, m>r$. The assumptions of Theorem 3.15 are valid for

$d \mu_{\xi_{n}}=\frac{1}{\left(\sqrt{\pi \xi_{n}}\right)^{N}} e^{-\left(\frac{\sum_{i=1}^{N} s_{i}^{2}}{\tilde{\xi} n}\right)} d s_{1} \ldots d s_{N}$ and $f_{j}$. Call $\gamma=0, \beta$. Let $f_{j,(\gamma+\alpha)} \in L_{p}\left(\mathbb{R}^{N}\right),|\alpha|=m, x \in \mathbb{R}^{N}$, and $p, q>1: \frac{1}{p}+\frac{1}{q}=1, \xi_{n} \in(0,1], n \in \mathbb{N}$. For $\widetilde{j}=1, \ldots, m$, and $\alpha:=\left(\alpha_{1}, \ldots, \alpha_{N}\right), \alpha_{i} \in \mathbb{Z}^{+}$, $i=1, \ldots, N,|\alpha|:=\sum_{i=1}^{N} \alpha_{i}=\widetilde{j}$, call

$$
\widetilde{c}_{\alpha, n, \tilde{j}}:=\frac{1}{\left(\sqrt{\pi \xi_{n}}\right)^{N}} \int_{\mathbb{R}^{N}} \prod_{i=1}^{N} s_{i}^{\alpha_{i}} e^{-\frac{\left(\sum_{i=1}^{N} s_{i}^{2}\right)}{\tilde{\xi} n}} d s_{1} \ldots d s_{N} .
$$

Then

$$
\begin{gathered}
\left\|\left(W_{r, n}^{[m]}(f ; x)\right)_{\gamma}-f_{\gamma}(x)-\sum_{\widetilde{j}=1}^{m} \delta_{\tilde{j}, r}^{[m]}\left(\sum_{|\alpha|=\widetilde{j}} \frac{\widetilde{c}_{\alpha, n, \tilde{j}} f_{\gamma+\alpha}(x)}{\prod_{i=1}^{N} \alpha_{i} !}\right)\right\|_{p, x} \\
\leq\left(\frac{2}{\sqrt{\pi}}\right)^{\frac{N}{p}}\left(\frac{m}{(q(m-1)+1)^{\frac{1}{q}}}\right)\left[(1+N)^{r p}+2^{r p} \Gamma^{N}((m+r) p+1,1)\right]^{\frac{1}{p}}
\end{gathered}
$$




$$
\left(\sum_{|\alpha|=m} \frac{1}{\prod_{i=1}^{N} \alpha_{i} !}\right)\left(\sqrt{\xi_{n}}\right)^{(m-r)}\left[\omega_{r}\left(f_{1, \gamma+\alpha}, \xi_{n}\right)_{p}+\omega_{r}\left(f_{2, \gamma+\alpha}, \xi_{n}\right)_{p}\right] .
$$

Proof. By Theorems 3.15, 3.19.

We give also

Theorem 3.35. Let $f: \mathbb{R}^{N} \rightarrow \mathbb{C}: f=f_{1}+i f_{2}, j=1,2$. Here $f_{j} \in C^{l}\left(\mathbb{R}^{N}\right), l, N \in \mathbb{N}$. The assumptions of Theorem 3.15 are valid for

$d \mu_{\xi_{n}}=\frac{1}{\left(\sqrt{\pi \xi_{n}}\right)^{N}} e^{-\left(\frac{\sum_{i=1}^{N} s_{i}^{2}}{\xi_{n}}\right)} d s_{1} \ldots d s_{N}$ and $f_{j}$. Call $\gamma=0, \beta$. Let $f_{j, \gamma} \in L_{p}\left(\mathbb{R}^{N}\right), x \in \mathbb{R}^{N} ; p, q>1$ : $\frac{1}{p}+\frac{1}{q}=1, \xi_{n} \in(0,1], n \in \mathbb{N}$. Then

1)

$$
\begin{gathered}
\left\|\left(W_{r, n}^{[0]}(f)\right)_{\gamma}-f_{\gamma}\right\|_{p} \leq \\
\left(\frac{2}{\sqrt{\pi}}\right)^{\frac{N}{p}}\left[(1+N)^{r p}+2^{r p} \Gamma^{N}(r p+1,1)\right]^{\frac{1}{p}} \frac{\left[\omega_{r}\left(f_{1, \gamma}, \xi_{n}\right)_{p}+\omega_{r}\left(f_{2, \gamma}, \xi_{n}\right)_{p}\right]}{\left(\sqrt{\xi_{n}}\right)^{r}} .
\end{gathered}
$$

2) Let $r \in \mathbb{N}-\{1\}, 0<\alpha \leq 1$, with $\omega_{r}\left(f_{j, \gamma}, t\right)_{p} \leq K t^{r-1+\alpha}, \forall t>0, K>0$ independent of $t$, $j=1$, 2. In this case

$$
\frac{\omega_{r}\left(f_{j, \gamma}, \xi_{n}\right)_{p}}{\left(\sqrt{\xi_{n}}\right)^{r}} \leq K \xi_{n}^{\left(\frac{r}{2}+\alpha-1\right)}, j=1,2
$$

As $n \rightarrow+\infty$ and $\xi_{n} \rightarrow 0$, then $\left(W_{r, n}^{[0]}(f)\right)_{\gamma} \stackrel{\|\cdot\|_{p}}{\rightarrow} f_{\gamma}$.

Proof. By Theorems 3.15 and 3.20.

We continue with

Theorem 3.36. Let $f: \mathbb{R}^{N} \rightarrow \mathbb{C}: f=f_{1}+i f_{2}, j=1,2$. Here $f_{j} \in C^{l}\left(\mathbb{R}^{N}\right), l, N \in \mathbb{N}$. The assumptions of Theorem 3.15 are valid for

$d \mu_{\xi_{n}}=\frac{1}{\left(\sqrt{\pi \xi_{n}}\right)^{N}} e^{-\left(\frac{\sum_{i=1}^{N} s_{i}^{2}}{\xi n}\right)} d s_{1} \ldots d s_{N}$ and $f_{j}$. Call $\gamma=0, \beta$. Let $f_{j, \gamma} \in L_{1}\left(\mathbb{R}^{N}\right), x \in \mathbb{R}^{N}, \xi_{n} \in(0,1]$, $n \in \mathbb{N}$. Then

1)

$$
\left\|\left(W_{r, n}^{[0]}(f)\right)_{\gamma}-f_{\gamma}\right\|_{1} \leq
$$




$$
\left(\frac{2}{\sqrt{\pi}}\right)^{N}\left[(1+N)^{r}+2^{r}\left(\frac{\lfloor e r !\rfloor}{e}\right)^{N}\right] \frac{\left[\omega_{r}\left(f_{1, \gamma}, \xi_{n}\right)_{1}+\omega_{r}\left(f_{2, \gamma}, \xi_{n}\right)_{1}\right]}{\left(\sqrt{\xi_{n}}\right)^{r}} .
$$

2) Let $r \in \mathbb{N}-\{1\}, 0<\alpha \leq 1$, with $\omega_{r}\left(f_{j, \gamma}, t\right)_{1} \leq K t^{r-1+\alpha}, \forall t>0, K>0$ independent of $t$, $j=1$, 2 . In this case

$$
\frac{\omega_{r}\left(f_{j, \gamma}, \xi_{n}\right)_{1}}{\left(\sqrt{\xi_{n}}\right)^{r}} \leq K \xi_{n}^{\left(\frac{r}{2}+\alpha-1\right)}, j=1,2
$$

and as $n \rightarrow \infty$ and $\xi_{n} \rightarrow 0$, we get $\left(W_{r, n}^{[0]}(f)\right)_{\gamma} \stackrel{\|\cdot\|_{1}}{\rightarrow} f_{\gamma}$.

Proof. By Theorems 3.15 and 3.21.

We finish with

Theorem 3.37. Let $f: \mathbb{R}^{N} \rightarrow \mathbb{C}: f=f_{1}+i f_{2}, j=1$, 2. Here $f_{j} \in C^{m+l}\left(\mathbb{R}^{N}\right), m, l, N \in \mathbb{N}$. The assumptions of Theorem 3.15 for $d \mu_{\xi_{n}}=\frac{1}{\left(\sqrt{\pi \xi_{n}}\right)^{N}} e^{-\left(\frac{\sum_{i=1}^{N} s_{i}^{2}}{\xi n}\right)} d s_{1} \ldots d s_{N}$ and $f_{j}$ are valid. Call $\gamma=0, \beta$. Let $f_{j,(\gamma+\alpha)} \in L_{1}\left(\mathbb{R}^{N}\right),|\alpha|=m, x \in \mathbb{R}^{N}, \xi_{n} \in(0,1], n \in \mathbb{N}$. For $\tilde{j}=1, \ldots, m$, and $\alpha:=\left(\alpha_{1}, \ldots, \alpha_{N}\right)$, $\alpha_{i} \in \mathbb{Z}^{+}, i=1, \ldots, N,|\alpha|:=\sum_{i=1}^{N} \alpha_{i}=\widetilde{j}$, call

$$
\widetilde{c}_{\alpha, n, \tilde{j}}:=\frac{1}{\left(\sqrt{\pi \xi_{n}}\right)^{N}} \int_{\mathbb{R}^{N}} \prod_{i=1}^{N} s_{i}^{\alpha_{i}} e^{-\frac{\left(\sum_{i=1}^{N} s_{i}^{2}\right)}{\xi_{n}}} d s_{1} \ldots d s_{N}
$$

Then

$$
\begin{gathered}
\left\|\left(W_{r, n}^{[m]}(f ; x)\right)_{\gamma}-f_{\gamma}(x)-\sum_{\tilde{j}=1}^{m} \delta_{\tilde{j}, r}^{[m]}\left(\sum_{|\alpha|=\tilde{j}} \frac{\widetilde{c}_{\alpha, n, \tilde{j}} f_{\gamma+\alpha}(x)}{\prod_{i=1}^{N} \alpha_{i} !}\right)\right\|_{1, x} \\
\leq\left(\frac{2}{\sqrt{\pi}}\right)^{N}\left(\sum_{|\alpha|=m}\left(\frac{1}{\prod_{i=1}^{N} \alpha_{i} !}\right)\left[\omega_{r}\left(f_{1, \gamma+\alpha}, \xi_{n}\right)_{1}+\omega_{r}\left(f_{2, \gamma+\alpha}, \xi_{n}\right)_{1}\right]\right) \\
\left(\sqrt{\xi_{n}}\right)^{m-r}\left[(1+N)^{r}+2^{r}\left(\frac{\lfloor e(m+r) !\rfloor}{e}\right)^{N}\right] .
\end{gathered}
$$

Proof. By Theorems 3.15 and 3.22. 


\section{References}

[1] G.A. Anastassiou, Rate of convergence of non-positive linear convolution type operators. A sharp inequality, J. Math. Anal. and Appl., 142 (1989), 441-451.

[2] G.A. Anastassiou, Moments in Probability and Approximation Theory, Pitman Research Notes in Math., Vol. 287, Longman Sci. \& Tech., Harlow, U.K., 1993.

[3] G.A. Anastassiou, Approximation by Multivariate Singular Integrals, Springer, New York, 2011.

[4] G. Anastassiou and S. Gal, Approximation Theory, Birkhaüser, Boston, Basel, Berlin, 2000.

[5] G. Anastassiou and R. Mezei, Uniform convergence with rates of smooth Gauss-Weierstrass singular integral operators, Applicable Analysis, 88, 7 (2009), 1015-1037.

[6] G.A. Anastassiou, R.A. Mezei, $L_{p}$ convergence with rates of smooth Gauss-Weierstrass singular operators, Nonlinear Studies, 17 (2010), no. 4, 373-386.

[7] I.S. Gradshteyn and I.M. Ryzhik, Table of Integrals, Series and Products, Eighth Edition, Elsevier, Amsterdam, New York, 2015.

[8] R.A. DeVore and G.G. Lorentz, Constructive Approximation, Springer-Verlag, Vol. 303, Berlin, New York, 1993. 